\title{
Fast-Neutron Coincidence-Counter Manual
}

N. Ensslin

T. L. Atwell*

D. M. Lee

B. Erkkila

R. S. Marshall

A. Morgan

C. Shonrock

B. Tippens

T. Van Lyssel 


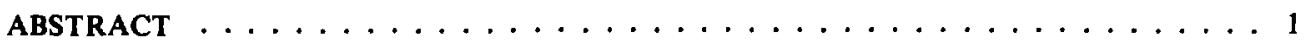

I. INTRODUCTION $\ldots \ldots \ldots \ldots \ldots \ldots \ldots \ldots \ldots \ldots \ldots \ldots$

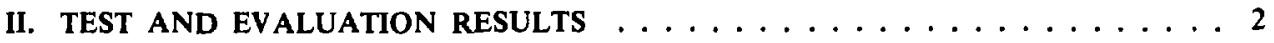

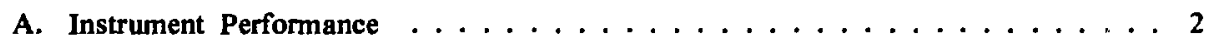

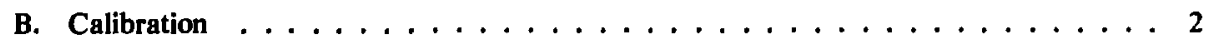

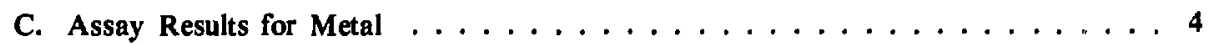

D. Assay Results for Residues $\ldots \ldots \ldots \ldots \ldots$

III. MECHANICAL DESCRIPTION $\ldots \ldots \ldots \ldots \ldots \ldots \ldots$

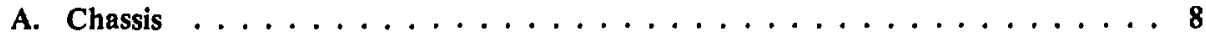

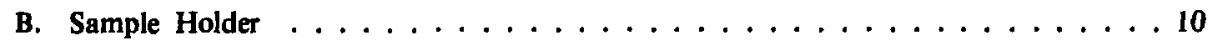

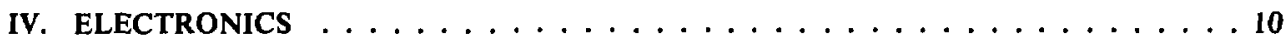

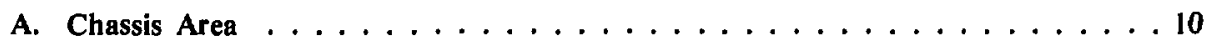

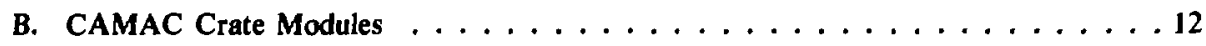

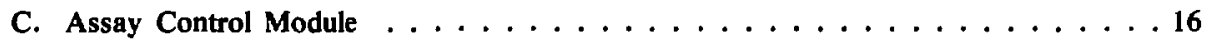

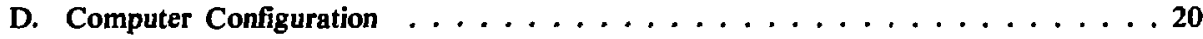

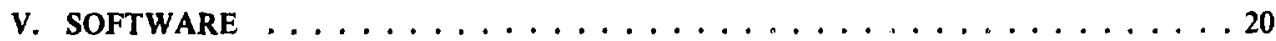

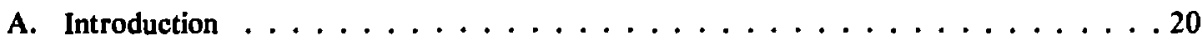

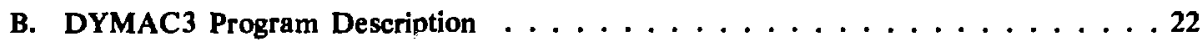

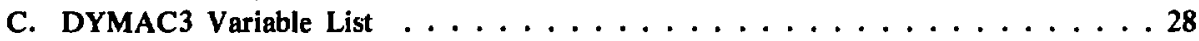

D. Calibration Constants for DYMAC3 as Stored in File RDINIT.D01 . . . . . . 30

E. Documentation for Least Squares Fit Program (LSQFIT) . . . . . . . . . 32

F. Additional Functions for MUBASIC . . . . . . . . . . . . . 34

VI. FAST ELECTRONICS TUNE-UP $\ldots \ldots \ldots \ldots \ldots \ldots \ldots \ldots \ldots \ldots$

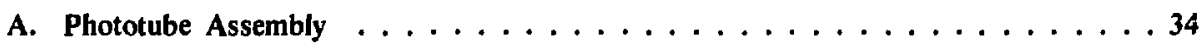

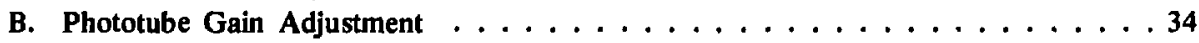

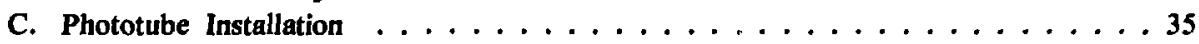

D. Phototube Gain and Timing Adjustment $\ldots \ldots \ldots \ldots \ldots \ldots$

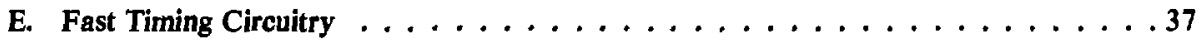

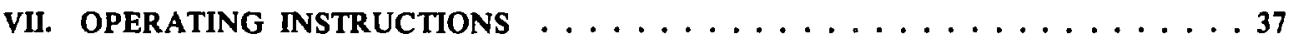

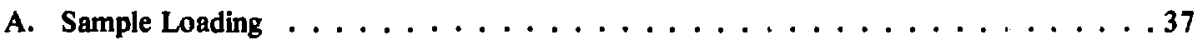

B. Tl 743 Silent 700 Terminal Settings . . . . . . . . . . . . . 38

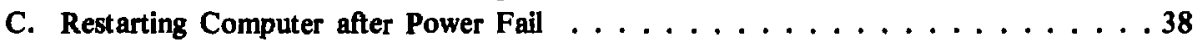

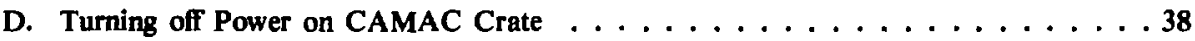

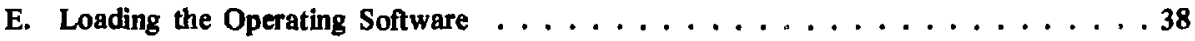

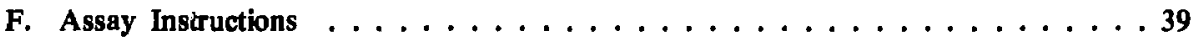

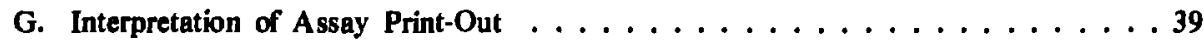

H. Measurement Control Procedures .................42

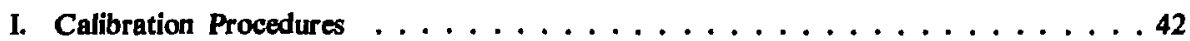




\title{
FAST NEUTRON COINCIDENCE, COUNTER MANUAL
}

\author{
by \\ N. Ensslin, T. L. Atwell, D. M. Lee, B. Erkkila, R. S. Marshall, A. Morgan, \\ C. Shonrock, B. Tippens, and T. Van Lyssel
}

\begin{abstract}
The fast neutron counter (FNC) described in this report is a computer-based assay system employing fast-pulse counting instrumentation. It is installed below a glove box in the metal electrorefining area of the Los Alamos National Laboratory Plutonium Processing Facility. The instrument was designed to assay plutonium salts and residues from this process and to verify the mass of electrorefined nietal. Los Alamos National Laboratory Groups Q-I, Q-3, and CMB-I 1 carried cut a joint test and evaluation plan of this instrument between May 1978 and May 1979. The results of that evaluation, a description of the FNC, and operating instructions for further use are given in this report.
\end{abstract}

\section{INTRODUCTION}

During the past several years the Los Alamos National Laboratory Plutonium Processing Facility has received a large amount of nondestructive assay (NDA) equipment as part of the dynamic materials accountability (DYMAC) safeguards program. In the metal electrorefining area, there was a need for an instrument that could assay the plutonium salts and fluoride residues produced in the electrorefining process and that could verify the impure metal input and the pure metal output of this process. It appeared that the Los Alamos-designed random driver might be appropriate for this problem. This instrument contains two strong AmLi random neutron sources that can induce fissions in the sample. When the sources are removed, the instrument passively measures coincidence neutrons from the spontaneous fission of the even isotopes of plutonium. If the plutonium is in the form of fluorides or other compounds with high $(a, n)$ cross sections, these neutrons may induce fissions that contribute to the coincidence signal. Then it is desirable to introduce the random neutron sources and actively interrogate the odd isotopes of plutonium. The net active-minus-passive coincidence response is proportional to the mass of ${ }^{239} \mathrm{Pu}$ and (hopefully) independent of chemical impurities.

For these reasons the fast neutron counter (FNC) consists of a random driver chassis with two AnLi neutron sources that can be moved in and out under computer control. The chassis contains three plastic scintillators to provide neutron multiplicity information on large multiplying samples. Lead shielding is used to reduce the flux of fission gamma rays to the scintillators. Fast-pulse processing circuits are used to reduce the background of accidental coincidences from the neutron sources and from impure samples and to further reduce the gamma-ray response. A Digital Equipment Corp. (DEC) PDP-11/04 computer is provided for assay control, source motion, and data analysis. 
The FNC was installed in the metal electrorefining area in May 1978 for a 1-yr test and evaluation period. The Safeguards Assay Group (Q-1) was responsible for installation, checkout, calibration, maintenance, and documentation. Group Q-3 assisted with these activities and provided a cognizant scientist to train operators, help carry out assays, and maintain a record of system performance. Group CMB-11 performed a series of assays of electrorefining residues and plutonium metal, including daily background and normalization checks. CMB-11 also provided (through $\mathrm{CMB}-1$ ) information on the isotopic composition of all samples and a complete destructive analysis of six residue samples.

Section II of this report describes the results of the test and evaluation of the FNC. Sections III, IV, and $\mathrm{V}$ describe the FNC chassis, electronics, and software. Instructions for tune-up, operation, and maintenance are given in Secs. VI, VII, and VIII.

\section{TEST AND EVALUATION RESULTS}

\section{A. Instrurnent Performance}

During the test and evaluation period, the FNC functioned well, with only a few days lost to equipment repair or replacement. In particular, the mechanism for moving the AmLi interrogation sources into place for active assays was reliable. In the year following the test and evaluation period, failures of individual channels of the 4-, 8-, or 12-channel nuclear instrument modules (NIM) were more frequent. Exchange and repair of the affected modules resulted in several weeks of lost time. Some repair of computer peripherals and motor controllers and microswitches was also required. Part of this damage was the result of improper handling by plant workmen engaged in other duties.

The temperature correction originally applied to the data was deleted because of the stable plant environment. A correction for moderation of interrogation neutrons by the sample-as measured by the flux of thermal neutrons recorded in two ${ }^{3} \mathrm{He}$ detectors-was also found to be unnecessary for the small sample sizes encountered in practice. Both the temperature correction and the flux correction were replaced by alarm limits. A correction for sample fill height also was not needed, because the integrated response of the instrument is uniform to within several per cent over the range of sample sizes.

Sample assays were corrected only for background, detector efficiency, and self-multiplication within the sample. Coincidence background rates ranged between 3 and 10 counts/s, depending on the amount of material in the vicinity. The detector efficiency (whose absolute value is about $17 \%$ ) was stable to $\pm 1 \%$ of the nominal value for 1 -wk periods and has been stable to $\pm 2 \%$ since installation. This stability is somewhat better than that reported for earlier random drivers and is presumably due to the stable temperature in the plant. The correction for self-multiplication is based on the ratio of triple to double coincidences, as described in Sec.V.B. The formulas described there also provide for samples that emit neutrons produced in $(\alpha, n)$ reactions.

\section{B. Calibration}

After the FNC was installed in the plant, a variety of well-known plutonium metal, oxide, and fluoride samples were run in the FNC to provide data for calibration curves. When measurements of process material began, some adjustments of the calibration carves and correction factors were required. At present, the instrument is calibrated for five classes of material: oxides, fluorides, pure electrorefined metal, impure metal from oxide reduction, and electrorefining residues. All calibration curves are of the form Mass $=\mathrm{A}($ Response $) /[1+\mathrm{B}$ (Response) $]$. The curves are nearly linear ( $\mathrm{B}$ is small) because of the self-multiplication correction. One self-multiplication correction is used for all passive assays, another for all active assays. 
The oxide calibration was based on oxide standards measured after installation. Two curves were provided, one for passive assays (Fig. 2-1) and one for active assays (Fig. 2-2). An active assay is automatically carried out by the computer if the passive assay indicates that the sample may be impure [that is, unusually high singles count rate resulting from $(\alpha, n)$ reactions]. Because impurities can only increase the measured response, the computer then selects the minimum result as the final assay. On the basis of the calibration, the FNC should provide oxide assays of about $2 \%$ accuracy, but no oxides have been assayed yet.

For plutonium fluorides, only a passive calibration (not illustrated) was provided. This should provide assays of pure $\mathrm{PuF}_{4}$ to $\sim 5 \%$ accuracy, but so far no fluorides have been assayed. An active calibration was not available at the time of the evaluation because of a saturation problem in the electronics. This problem has now been diagnosed as an imbalance between the double and accidental double coincidence circuits. The imbalance has been removed by the addition of clipping lines to define the gate lengths precisely. It should now be possible to calibrate the $\mathrm{FNC}$ for active assays of $\mathrm{PuF}_{4}$ up to about $800 \mathrm{~g}$. However, the high count rate observed with this material (about 1000 counts/s.g) will limit the active assay precision for such large samples to about $10 \%$ in a 1000 -s counting time.

Figure 2-3 shows the FNC calibration curve for electrorefined metal ( $>\$ 9.9 \%$ purity). Pure metal required only a passive measurement. The calibration curve is the same, within statistical errors, as the passive oxide calibration curve. The metal consisted of irregular curved pieces stacked in a small can. As a consequence of the self-multiplication correction, the response was nearly independent of the geometrical configuration. Each data point was measured with $\sim 1 \%$ statistical precision. The actual scatter of $2.5 \%(1 \sigma)$ is attributed primarily to the approximations inherent in the self-multiplication correction.

Figure 2-4 shows thi, FNC calibration for impure metal, which required an active assay. This material consisted of $600 \mathrm{~g}$ metal buttons, derived from oxide reduction, which contained 10 to $20 \mathrm{~g}$ of impurities and 1 to $2 \mathrm{~g}$ of magnesium or calcium salts acquired during reduction. Induced fissions from $(\alpha, \mathrm{n})$ reactions in the salts make the passive assay high by 100 to $200 \mathrm{~g}$. The active assay is more accurate, but is subject to $3 \%$ statistics resulting from the active-minus-passive difference measurement. The active metal and active oxide calibrations both curve downward with increasing mass and are not the same within statistical errors. Probably this is due to absorption of interrogating neutrons by the samples.

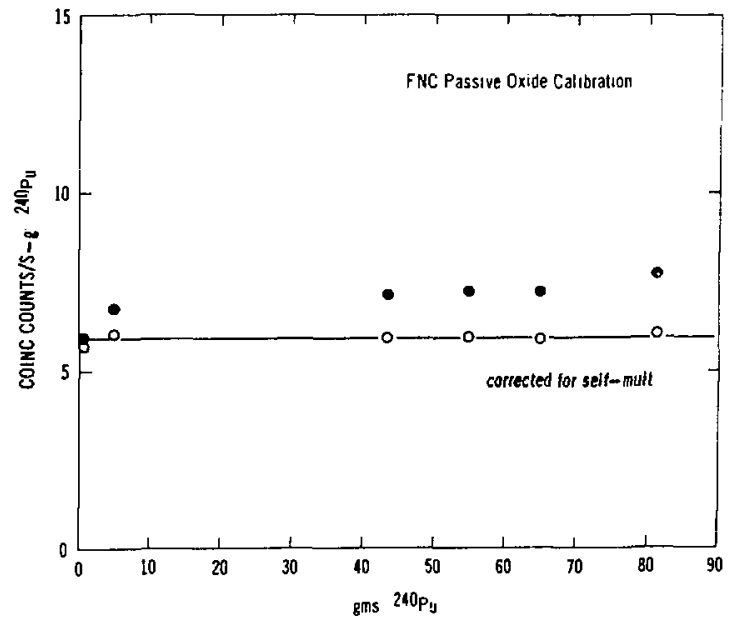

Fig. 2-1.

Data used for FNC passive oxide calibration. The calibration was based on the selfmultiplication-corrected values.

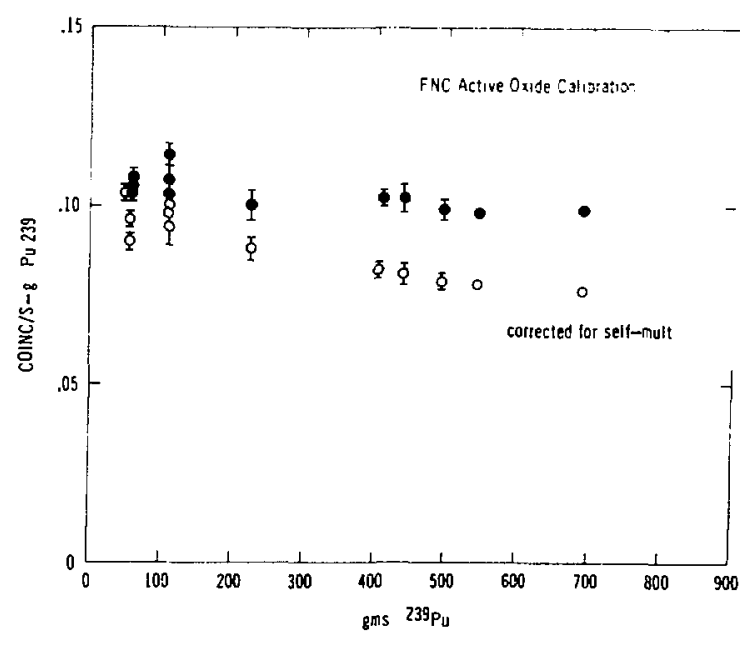

Fig. 2-2.

Data used for FNC active oxide calibration. The calibration was based on the selfmultiplication-corrected values. 


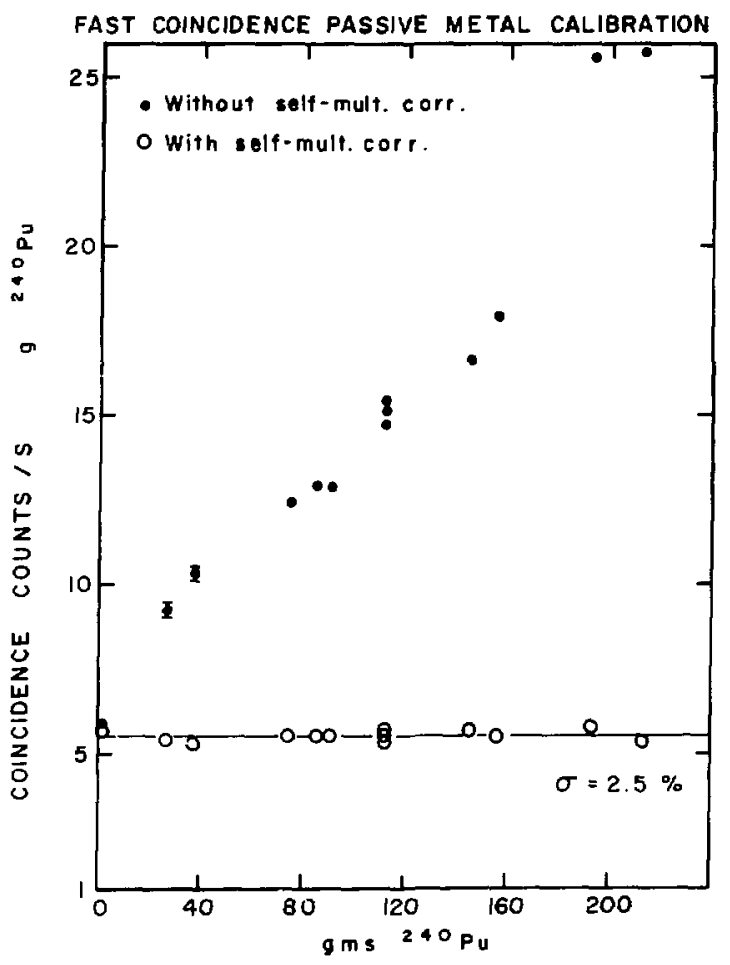

Fig. 2-3.

Data used for FNC pure metal calibration (passive only).

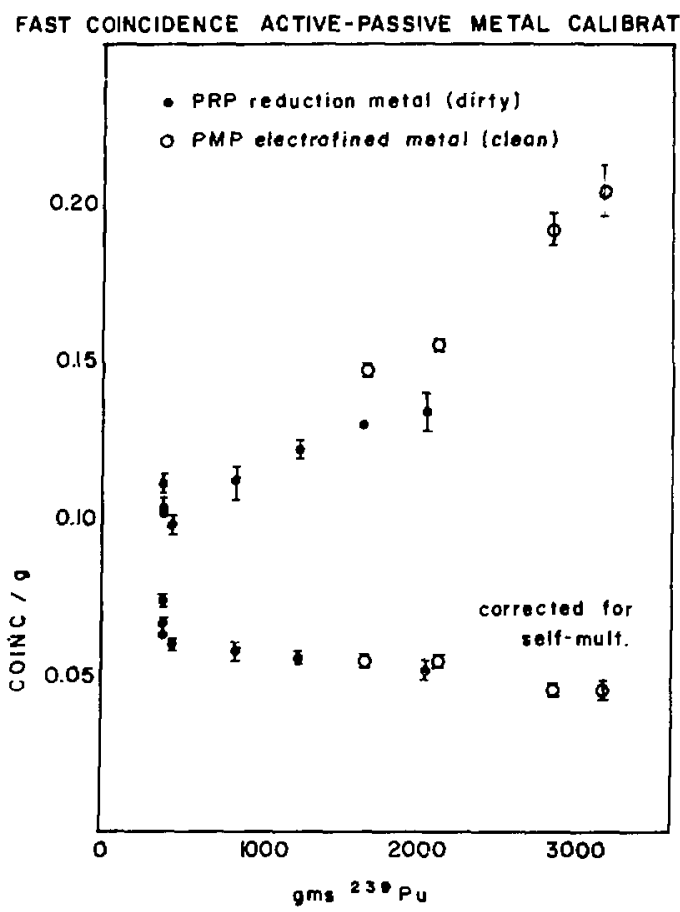

Fig. 2-4.

Data used for FNC impure metal calibration (active only). Both impure metal and electrorefined metal data were used, because both should show the same response to active interrogation.

For the electrorefining resiciues, the active and passive oxide calibration curves (Figs. 2-1 and 2-2) were used.

\section{Assay Results for Metal}

Table II- 1 summe: azes the results of the test and evaluation period for the five categories of material for which the FNC was calibrated. As mentioned earlier, no oxide or fluoride samples were assayed after calibration. For pure electrorefined metal, ...e FNC provided a verification with a random error of $3.5 \%$ of the measured weight. The assays were $3.1 \%$ high (average), calling for some adjustment of the calibration curve. The statistical precision of each assay was about $1 \%$ in a 500 -s counting time, but the self-multiplication correction facto: was 2 to 3 . Because of the magnitude of this approximate correction, the observed precision was worse than the statistical precision. Also, the error obtained at the time of the assay was somewhat worse than 3 to $4 \%$ because the operator usually did not know the isotopic composition of the sample. The actual effective ${ }^{240} \mathrm{Pu}$ was typically 1 to $2 \%$ different from the nominal value of $6 \%$.

For impure metal cbtained frolı oxide reduction, the FNC provided a verification with a random error of $3.7 \%$ of the by-difference value. This error is due almost entirely to the 3 to $4 \%$ statistical precision of the active assay (500-s counting time). The FNC assays averaged $5.2 \%$ lower than the measured weight of the sample. After allowance for the impurities in the metal, these assays averaged $2.2 \%$ lower than the by-difference values. A.gain, an adjustment to the calibration curve would eliminate this bias. 
TABLE II-1

TEST AND EVALUATION RESULTS

\begin{tabular}{|c|c|c|c|c|c|}
\hline $\begin{array}{l}\text { Sample } \\
\text { Category }\end{array}$ & $\begin{array}{l}\text { Assay } \\
\text { Type }\end{array}$ & $\begin{array}{l}\text { Source of } \\
\text { Calibration }\end{array}$ & $\begin{array}{l}\text { Number of Samples } \\
\text { Since Calibration }\end{array}$ & $\begin{array}{c}\text { Systematic Error } \\
\text { (with respect to by-diff) } \\
\%\end{array}$ & 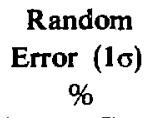 \\
\hline oxide & $\begin{array}{l}\text { passive } \\
\text { (active) }\end{array}$ & oxide standards & none & & \\
\hline fluoride & passive & $\mathrm{PuF}_{4}$ powder & none & & \\
\hline $\begin{array}{l}\text { electrorefined } \\
\text { metal }\end{array}$ & passive & metal weight & 10 & +3.1 & 3.5 \\
\hline impure metal & active & $\begin{array}{l}\text { active assay of } \\
\text { electrorefined } \\
\text { metal }\end{array}$ & 44 & $-\quad 2.2$ & 3.7 \\
\hline residues & $\begin{array}{c}\text { active } \\
\text { (passive) }\end{array}$ & oxide standards & $\begin{array}{l}24 \\
24\end{array}$ & $\begin{array}{c}0 \\
+\quad 36.0\end{array}$ & $\begin{array}{l}15.0 \\
37.0\end{array}$ \\
\hline
\end{tabular}

For pure metal, the FNC assay was sess accurate than the electronic balance measurement. For impure metal, the FNC assay accuracy was comparable to (but not better than) the $3 \%$ difference between the measured and by-difference weight. Thus for both categories of metal, the FNC assays do not replace traditional accounting values, but do provide a verification of the amount of special nuclear material piesent.

\section{Assay Results for Residues}

The residues $\mathrm{Ci}^{-1}$ the electrorefining process contain $30 \mathrm{~g}$ of $\mathrm{PuF}_{3}$ (the converted catalyst), $1400 \mathrm{~g}$ of $\mathrm{KCl}$ and $\mathrm{NaCl}$ electrolytes, and small beads of impure plutonium. These electrorefining salts are strong $(\alpha, \mathrm{n})$ neutron emitters, and contain enough plutoniuin to be self-shielding with respect to gamma radiation and self-multiplying with respect to neutrons. The total mass of plutonium in the residues assayed by the FNC varied between 40 and $600 \mathrm{~g}$. (The three samples with values between 40 and $60 \mathrm{~g}$ represent abortive electrorefining runs that occurred during plant start-up.) To assay these residues, the active and passive oxide calibration curves were used.

Table II-1 and Fig. 2-5 summarize the results obtained for the active and passive residue assays. As expected, the passive assays were high and very inaccurate because of $(\alpha, n)$-induced fissions. This is why the active neutron technique was chosen. However, the active assay results were also disappointing. Although there is no systematic bias relative to the oxide standards, the 1-std-dev scatter between the active assays and the plant by-difference values was $15 \%$. The statistical precision of the assays was 3 to $5 \%$ for most of the residues, but was as large as $15 \%$ for the smallest samples. The accuracy of the plant by-difference values is not exactly known, but has been estimated from the values obtained when the residues were totally dissolved. Six residues were analyzed by CMB-1 as part of the test and evaluation 


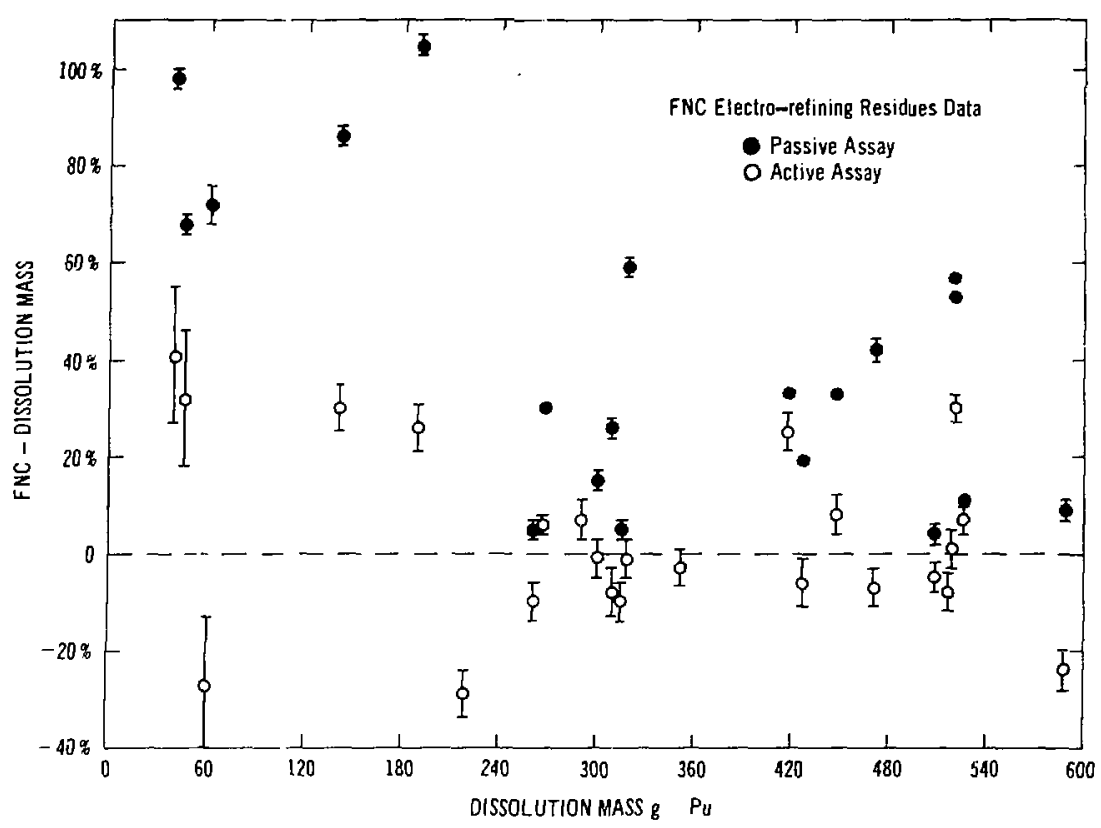

Fig. 2-5.

Comparison of FNC assays of electrorefining residues with dissolution values for the plutonium mass.

plan, and 10 others were reprocessed by CMB-11 during the past year. The average deviation between the original by-difference and the final dissolution value is $10 \%$. However, this deviation is not responsible for the scatter in the FNC assays. As shown in Fig. 2-5, the deviation between the FNC assays and the final dissolution values is still large and is still roughly $15 \%$.

One residue assay, which yielded a value of $333 \pm 14 \mathrm{~g}$, has been excluded from the above comparisons. The plant by-difference value was $189 \mathrm{~g}$. Because of the large discrepancy, this sample was submitted to CMB-1 for complete destructive analysis, with a result of $349 \mathrm{~g}$. After this result was obtained, a check of the plant by-difference calculation showed that an incorrect impurity factor had been used in the calculation. Thus the FNC residue assays are able to detect large discrepancies in the by-difference values, although the average accuracy is only about $15 \%$.

In an attempt to improve this accuracy, the residue data were reanalyzed in several different ways. The dissolution value (when available) or the plant by-difference value was taken to be the true mass. The goal was to find a correction that would make the observed active coincidence response/g of ${ }^{239} \mathrm{Pu}$ nearly constant from sample to sample. The following observations were made.

1. There is no correlation between the active double coincidence response/g and the passive triple/double coincidence ratio. The triples/doubles ratio is used to estimate and correct for sample self-multiplication. The lack of correlation implies that the self-multiplication correction is not effective for this material. This appears to be due to the large variation in the ratio of $(a, n)$ neutrons to spontaneous-fission neutrons. This ratio, which is $\mathbf{0}$ for metal and roughly 0.6 for oxide, varied between 0.3 and 9.8 for normal residues. For the three residues from the aborted electrorefining runs, the ratio was 30 to 33 . These three residues consisted almost entirely of $\mathrm{PuF}_{4}$ and the electrolytic salts.

2. There is no correlation between the active double coincidence response/g and the passive singles response/g. The passive singles response provides a means for estimating the ratio of $(\alpha, n)$ neutrons to spontaneous-fission neutrons. The lack of correlation again makes it difficult to apply a 
self-multiplication correction to the data. It does seem, however, that appreciable self-ri ultiplication is taking place in the residues. Most samples that show a high active coincidence response/g also have either a high triples/dosibles ratio or a high singles response/g. The self-multiplication correction available at this time, however, only reduces the observed scatter from $20 \%$ to $15 \%$.

3. There is some evidence that the gamma sensitivity of the plastic scintillators is affecting the assay accuracy. Table II-2 summarizes the passive FNC response to four oxide standards. STD-2 and STD-10 are pure oxide, and STD-5 and STD-7 contain MgO. The double coincidence response $/ \mathrm{g}$ is einhanced whenever the singles response/g is high. The triples response/g is unaffected within statistical errors. One explanation for these results is the presence of ( $\alpha, n$-gamma) or ( $\alpha, p$-gamma) reactions, where the neutron or proton and the gamma ray can be detected in coincidence. The reaction gamma rays are typically $1-2 \mathrm{MeV}$ in energy and have transmission factors of about $12 \%$ through the lead shielding (including downscattering effects). An examination of $\mathrm{PuF}_{4}$ and $\mathrm{PuO}_{2}$ (with $\mathrm{MgO}$ ) standards with a germanium detector revealed the presence of $1275-\mathrm{keV}$ gamma rays from the ${ }^{19} \mathrm{~F}(\alpha, \mathrm{p}){ }^{22} \mathrm{Ne}$ reaction and $1779-\mathrm{keV}$ gamma rays from the ${ }^{25} \mathrm{Mg}(\alpha, n){ }^{28} \mathrm{Si}$ reaction, respectively. The magnitude of the gamma-ray response was of the right order to explain the results in Table II-2. Although reaction gamma rays do not affect the active assay, they affect the calculation of the active self-multiplication correction, which is based on the passive singles. doubles, and triples response. Cousequently, some attempts were made to adjust the self-multiplication correction to allow for the gamma response of the FNC. These attempts have not been successful yet. Because the gamma emission rate of the residues depends on the impurity composition and is subject to severe attenuation effects, it may be too variable to permit a successful correction.

Thus the present accuracy of the FNC for the residue samples is still 15\%. This accuracy is thought to be limited by the gamma response of the scintillators. Future attempts to obtain better assays of electrorefining residues might include the following.

1. Assay a new series of residues with the present instrument for 1-to 3-h counting times. This would eliminate statistical precision as a factor influencing the evaluation of the assay accuracy. This new series of assays should include only residues from completed production runs for which final dissolution values are obtained. This would eliminate unusual electrorefining runs and errors in plant by-difference values as factors influencing the evaluation. These steps probably would yield accuracies better than $15 \%$, but not better than $5 \%$.

2. Base the assay on the passive triple coincidence response to reduce the effect of reaction gamma rays. This attempt would require some increase in the FNC sensitivity to get better statistical precision. Also, a multiplication correction would still be needed, and this correction would still require meaningful single and double coincidence data.

3. Increase the amount of lead between the sample well and the plastic scintillators from 2 in. to 3 in. or more to reduce the effect of reaction gamma rays. This would require rebuilding the FNC chassis.

TABLE II-2

\section{OXIDE STANDARDS IN FNC}

\begin{tabular}{lrrrr} 
Sample & Mass & Singles $/ g$ & Doubles $/ g$ & Triples $/ g$ \\
\hline & & & & \\
STD-2 & 5 & $356 \pm 56$ & $6.74 \pm 0.18$ & $0.082 \pm 0.018$ \\
STD-5 & 60 & $1429 \pm 36$ & $12.23 \pm 0.04$ & $0.065 \pm 0.003$ \\
STD-10 & 60 & $318 \pm 3$ & $6.72 \pm 0.03$ & $0.074 \pm 0.005$ \\
STD-7 & 120 & $039 \pm 16$ & $10.40 \pm 0.16$ & $0.073 \pm 0.002$
\end{tabular}


4. Assay a series of residues with a thermal neutron counter to evaluate its suitability for this category of material. Although electronic deadtime and induced-fission self-multiplication effects are known to be severe, the insensitivity of the ${ }^{3} \mathrm{He}$ tubes to gamma rays may be more important. Eight of the residues analyzed for this report were also assayed with a thermal neutron counter. The results (Table II-3) were of comparable accuracy with the FNC assays, implying that this technique deserves further study.

5. Initiate research on improved self-multiplication corrections or on a fast detector that is insensitive to gamma rays (such as a liquid scintillator with pulse shape discrimination circuitry). Such research might lead to more accurate assays at some future time.

6. Lastly, it should be noted that good assays of residues have been obtained with calorimetry if the Americium content is well known and that good results have been obtained with segmented gamma scanning techniques if the residues are pulverized and do not contain many beads of metal. ${ }^{1}$

\section{Iil. MECHANICAL DESCRIPTION}

\section{A. Chassis}

Figure 3-1 is a top view of the FNC chassis. This chassis is mounted beneath a glove box, as illustrated in Fig. 3-2. The chassis can be separated into two parts along the dashed line in Fig. 3-1. The front part of the chassis is a $U$-shaped assembly consisting of three plastic scintillators that are surrounded by 2 in. of lead on the interior and $1 \mathrm{in}$. elsewhere. The interior of the $U$ is a $6-\mathrm{mm}$ boral-lined cavity that surrounds the sample well protruding from: underneath the glove box. The boral hardens the neutron spectrum, and the lead shielding reduces the gamma background in the scintillators. The front assembly is mounted on four large caster wheels. It can be rolled into place beneath the glove box and bolted to the steel back support frame.

This back support frame is permanently bolted to the floor. It supports the steel and nickel neutron source reflectors, the neutron source cable mechanism, and two 8-in. active-length ${ }^{3} \mathrm{He}$ tubes, which can be used as thermal neutron monitors. For active assays, the AmLi neutron source is raised from beneath the floor by a Teleflex cable, pulleys, and a dc motor. The motor is visible in Fig. 3-2 to the right of the

TABLE II-3

FIRST RESIDUES DATA

\begin{tabular}{rrrrc}
$\begin{array}{c}\text { Sample } \\
\text { ID }\end{array}$ & $\begin{array}{c}\text { By-Diff } \\
\text { Mass }\end{array}$ & $\begin{array}{c}\text { Destructive } \\
\text { Analy sis }\end{array}$ & $\begin{array}{c}\text { FNC } \\
\text { Active }\end{array}$ & $\begin{array}{c}\text { TNC } \\
\text { Passive }\end{array}$ \\
\hline & & & & \\
XBL-300 & 422 & 426.0 & $402+20$ & 464 \\
301 & 474 & 448.0 & $482 \pm 18$ & $479 \pm 14$ \\
100 & 268 & 267.0 & $284 \pm 4$ & $263 \pm 32$ \\
302 & 557 & 526.0 & $562 \pm 20$ & $542 \pm 27$ \\
101 & 42 & 40.4 & $57 \pm 8$ & \\
102 & 50 & 44.0 & $58 \pm 8$ & $16 \pm 22$ \\
303 & 466 & 416.0 & $518 \pm 19$ & $516 \pm 35$ \\
304 & 300 & - & $298 \pm 13$ & $283 \pm 28$ \\
103 & 53 & 60.0 & $44 \pm 6$ & $77 \pm 26$ \\
\hline
\end{tabular}




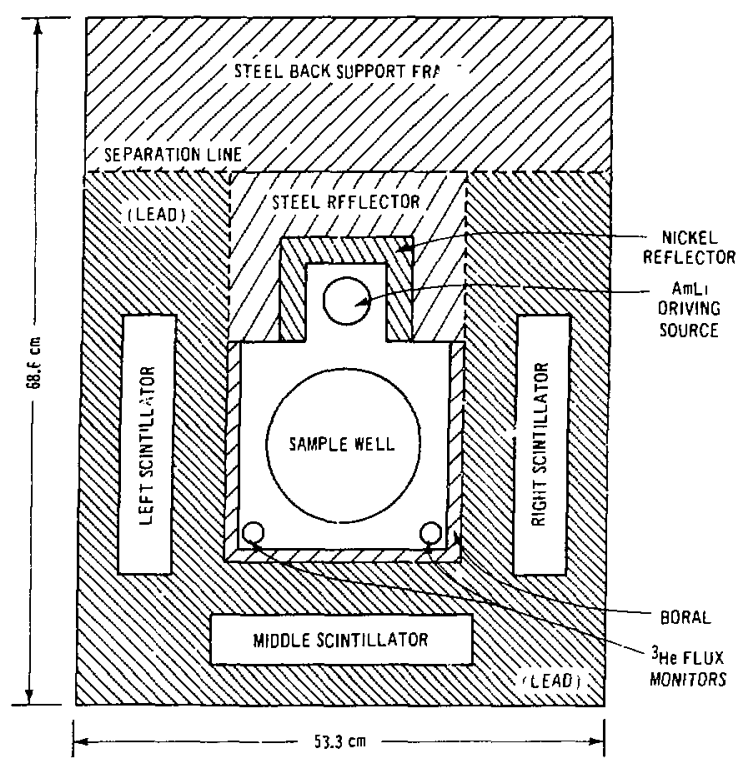

Fig. 3-1.

Top view of the FNC detector chassis.

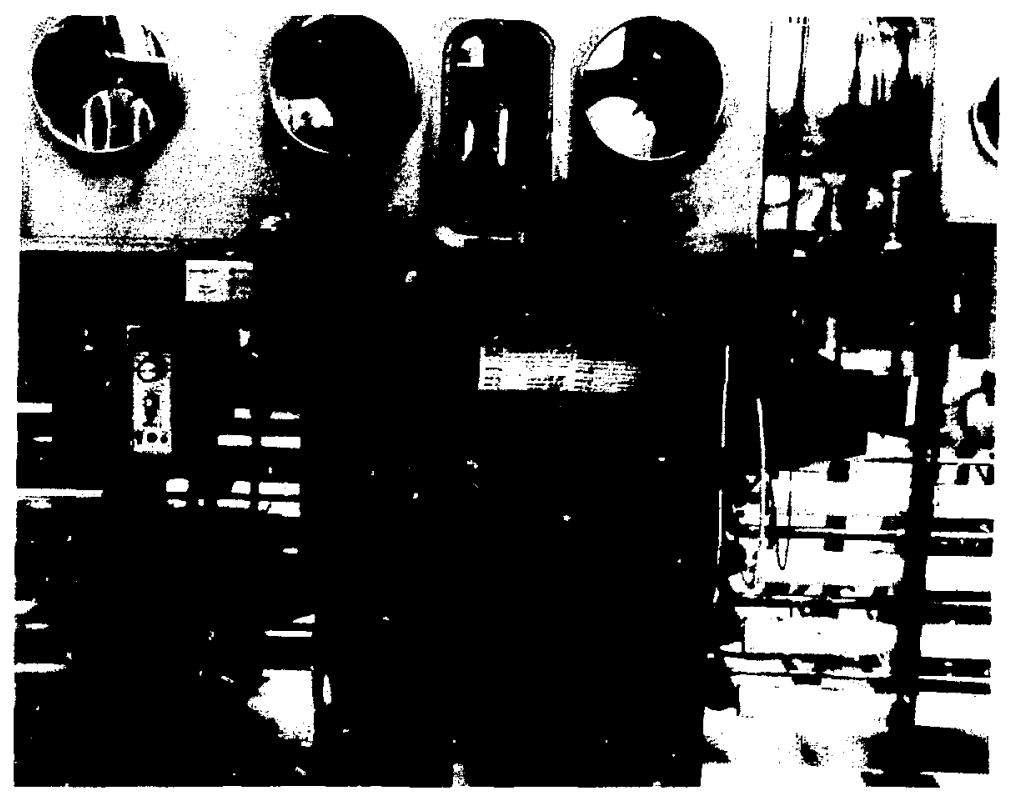

Fig. 3-2.

Location of the FNC detector chassis beneath a transfer glove box (drop box) in the Los Alamos Plutonium Processing Facility. 
chassis. It is a Bodine NSH-34Rd right-angle dri\%e motor with $142 \mathrm{lb}$-inches of torque, identical to those used for the thermal neutron colnters elsewhere in the plant. Both parts of the FNC chassis togcither are about 54 by 59 by $90 \mathrm{~cm}$ high and weigh about $1400 \mathrm{~kg}$.

The three plastic scintillators are $5-$ by $25-$ by $60-\mathrm{cm}$ rectangular slabs of Pilot $F$ (Nuclear Enterprises, San Carlos, California) encased in aluminum. Pilot $F$ has a long light-attenuation length $(300 \mathrm{~cm})$ and a large light output (64\% of anthracene), making it suitable for this application. Each scintillator is viewed by two RCA 8575 photomultyplier tubes (PMTs), optically coupled with DOW Corning Corp. 20-057 optical coupling compound. Each phototube is coupled to an Ortec Inc. 265 base.

The two AmLi neutron sources were obtained from Monsanto Research Corp. MRC-65 has a strength of $4.93 \times 10^{5} \mathrm{n} / \mathrm{s}$, and MRC-66 has a strength of $4.87 \times 10^{5} \mathrm{n} / 3( \pm 3 \%)$. Each source contains about 2.4 $\mathrm{g}$ of $\mathrm{AmO}_{2}$ and $19 \mathrm{~g}$ of $\mathrm{LiH}_{2}$ and emits $8.1 \mathrm{Ci}$ of radiation. The sources are doubly encapsulated in stainless steel by the manufacturer. At Los Alanos, a 3/8-in.-thick tungsten shield was added, which reduces the dose caused by $60-\mathrm{keV}$ gamma ravs from americium by roughly 2 orders of magnitude.

When the two neutron sources are not in use, they are kept about $4 \mathrm{ft}$ below the chassis in a shielded cask that is mounted to the ceiling of the room below. The cask surrounds the sources with $3 / 4 \mathrm{in}$. of lead and 2 in. of polyethylene lined with cadmium on the outside. This additional shielding reduces the dose at the surface of the container to $1.5 \mathrm{mrad} / \mathrm{h}$. A raicroswitch inside this shielded cask allows the computer to determine when the sources are inside.

When the two neutron sources are in use, they are up agannst a second microswitch located at the top of the back support frame. The sources are centered with respect to the scintillators. The center line is 9 $3 / 4$ in. below the microswitch, and $163 / 4$ in. below the bottom of the glove box. The centers ci the sources are $103 / 8$ in. apart. With the sources in these positions, the integra'ed active response is constant to within $2 \%$ for either 4 -in.- or 11 -in.-high sample cans, provided they are at least half full. A similar statement holds true for the passive response of the detector, so that no fill-height correction is necessary.

\section{B. Sample Holder}

Figure 3-3 shows the holder that is used to load samples into the FNC from the glove box above. The floor of the sample holder is $41 / 2$ in. below the scintillator and neutron source center line. The holder is tall enough to accommodate il $3 / 8$ in.-high residue cans. When 4 -in.-high cans with buttons are assayed, a 3-in. spacer is used to center the sample. Above the sample cavity, the holder contains about 4 in. of aluminum and steel reflector material and a small Bodine KYC-22T5, Model No. 765, 3-rpm motor. This motor slowly rotates the sample during the assay. The entire sample holder mechanism is raised and lowered by another Bodine NSH-34Rd right-angle drive motor mounted above the glove box. As can be seen in Fig. 3-3, both the power cord for the rotator motor and the cable connection to the lifting motor can be disconnected from the sample holde: if utis glove box area is needed for other work.

Los Alamos Group Q-1 has the original assembly and detail drawings for the FNC chassis.

\section{ELECTRONICS}

Tahle IV-1 is a list of the electronic components for this irstrument. The layout and interconnection of thise components are described in this section. Tune-up instructions are given in Sec. VI.

\section{A. Chassis Area}

The high-voltage (HV) distribution box for the PMTs is mounted on the left side of the FNC chassis (Fig. 3-2). This box contains six $300 \mathrm{k} \Omega$ 10-turn potentiometers that receive high voltage on cable 7 from 


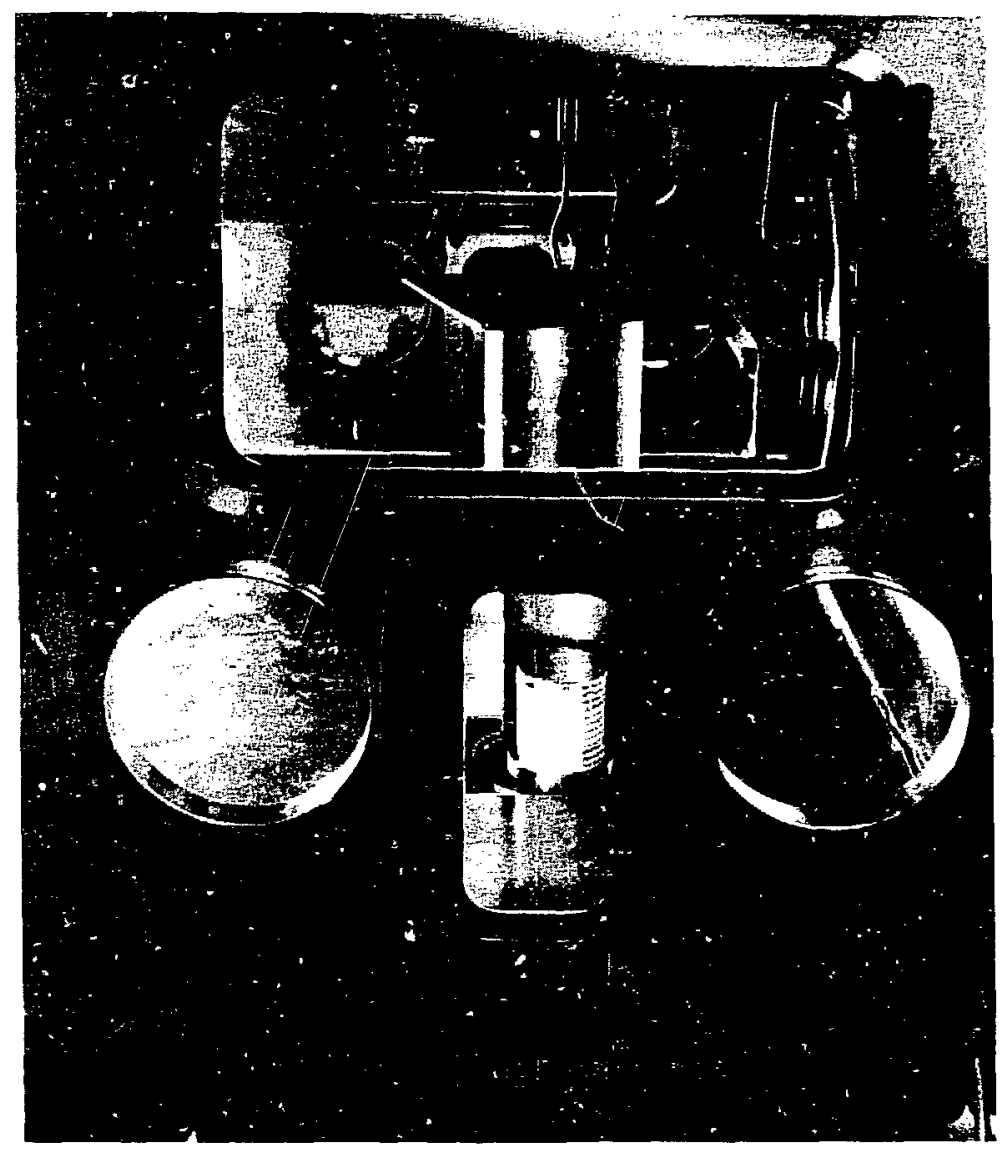

Fig. 3-3.

Sample holder mechanism visible through the glove box window.

the Power Designs HV supply. The potentiometers are used to form six voltage divider circuits that supply high voltage to the PMTs on cables 1 through 6. In this way the PMT gains can be individually adjusted to give equal magnitude output signals.

The junction box and preamplifier box for the ${ }^{3} \mathrm{He}$ tube flux monitor circuit are mounted behind and below the HV distribution box. Figure 4-I traces the signal path from the tubes to the CAMAC scaler. Figure 4-2 is a schematic of the Los Alamos-Jesigned preamplifier. ${ }^{3}$ With a tube high voltage of +1500 $\mathrm{V}$, the preamplifier output is typically $100-300 \mathrm{mV}, 70-80 \mu \mathrm{s}$ time constant. It is customary to adjust the amplifier gain (on the order of 50) to obtain 0- to 6-V unipolar output pulses.

Helium-3 detectors also detect (gamma,e) reactions in the gas, but the lower $\mathrm{dE} / \mathrm{dx}$ of the electrons means that these pulses will be wider in time. A relatively fast amplifier time constant of $0.5 \mu$ s or less is used to clip pulses caused by (gamma,e) reactions so they appear in the noise. With this time constant, the total width of the amplifier output pulses is 2 to $4 \mu \mathrm{s}$. The single-channel analyzer (SCA) discriminator is set to $3 \mathrm{~V}$ to reject noise pulses. The SCA output is $+5 \mathrm{~V}, 500 \mathrm{~ns}$ wide. The transistor to transistor logic(TTL)-to-NIM converter will convert this output to NIM standard pulses $(-800 \mathrm{mV})$.

Also mounted near the counter chassis is the request assay box. This is visible in Fig. 3-2 directly below the glove-box floor and to the left of the chassis. Just below and to the left of the request assay box is the Minarik motor controller for the sample-holder mechanism. Behind this Minarik controller is a 
TABLE IV-1

FNC ELECTRONICS LIST

A. Chassis

High-voltage distribution box

${ }^{3} \mathrm{He}$ tube junction box

${ }^{3} \mathrm{He}$ tube preamplifier box

6 RCA 8575 photomultiplier tubes

6 Ortec Inc. 265 tube bases

Request assay box

Minarik motor controller for AmLi neutron sources

2 YSI No. 44211 thermilinear components

B. Texas Instruments Inc. $\mathbf{7 4 3}$ desktop hardcopy terminal

C. Electronics cabinet (Fig. 4-3)

DEC RX-11 dual floppy disk and controller

Patch panel

SEC 1510 CAMAC crate

DEC PDP-11/04 MC computer

DEC dc $11 \mathrm{~A}-8 \mathrm{~F}$ unibus cable

2 YSI No. 44211 thermilinear components

Power Designs 3K-40 HV supply

Variac 1592 automatic voltage regulator

High-speed fan/blower

second Minarik box used to control the source drive motor. Because the source drive motor controller and the request assay box are interfaced to the CAMAC assay control module, their electronic schematics are given in Sec. IV.C.

\section{B. CAMAC Crate Modules}

Figure 4-3 shows the layout of the electronics cabinet. The CAMAC crate is located just below the patch panel. Table IV-2 lists the modules located in the CAMAC. crate in the order of their slot addresses. The first 14 adciresses are used by 7 commercial NIM modules to avoid the use of a separate NIM bin. The NIM modules receive their power from the CAMAC crate through seven Nuclear Electronics, Inc. Model 7092 NIM-to-CAMAC adapters. One of the NIM modules, the LRS 365 AL logic unit, also requires +0.8 and $-5.0 \mathrm{~V}$, which it receives from an internal power supply that operates on $120 \mathrm{Vac}$. However, the CAMAC crate does not supply ac power to this module. For this reason a separate ac power cord has been added to the rear of this module. Addresses 16 to 21 are used by five commercia! CAMAC modules. Addresses 22 and 23 are used by a Los Alamos-designed assay control module, which is described in Sec. IV.C.

The first five NIM modules constitute the fast timing sircuits that generate single, double, and triple events. Fig. 4-4 is a diagram of this circuit, including the final outputs to the 12-channel CAMAC scaler. The six inputs to the LRS 612 12-channel PMiT amplifier are the negative timing anode signals (50 $\Omega$ dc-coupled) from the Ortec 265 PMT bases. After amplification (x10) these signals are up to several volts in magnitude and 10 to $20 \mathrm{~ns}$ wide (see Fig. 6-1). The LRS 623 octal discriminator rejects PMT noise pulses, and after discrimination the pulses are set to 10 -ns width, NIM level $(-800 \mathrm{mV})$. 


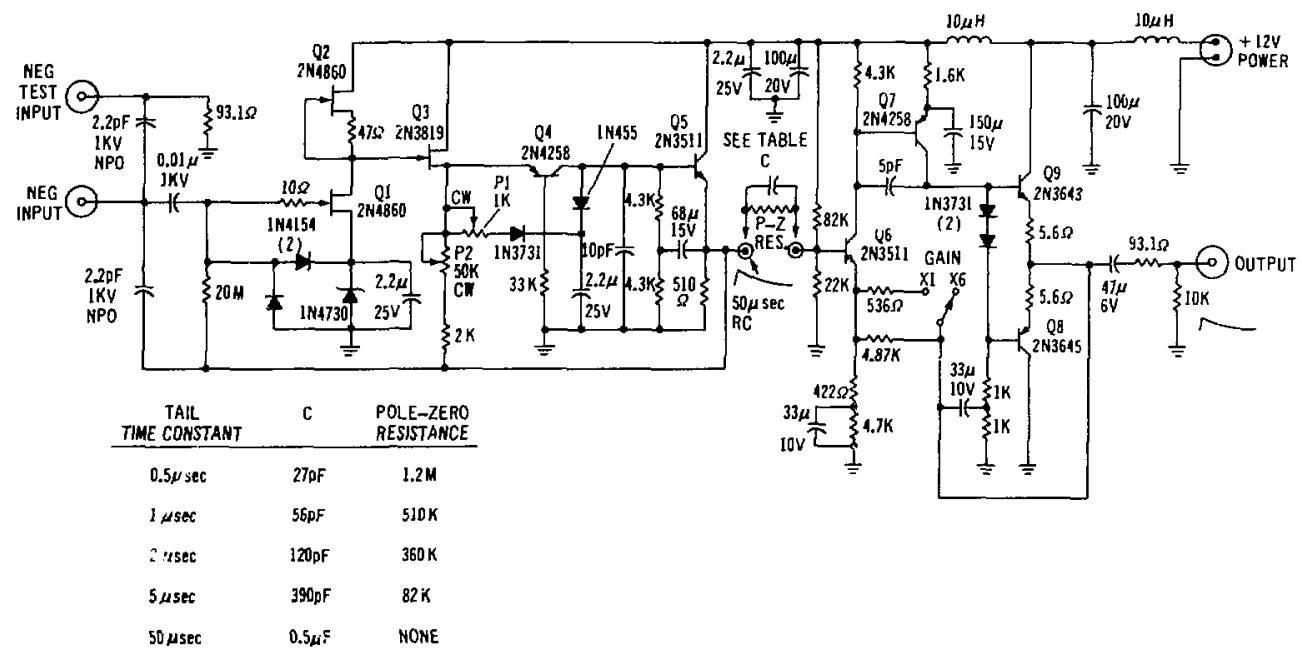

Fig. 4-2.

Schematic of Los Alamos-designed proportional counter preamplifier used for the ${ }^{3} \mathrm{He}$ tube flux monitor.

An LRS 622 quad coincidence unit is used to AND together the two PMT signals from each scintillator. This further reduces the amount of PMT noise entering the coincidence circuit and reduces the measured singles rate by roughly $50 \%$. It is also helpful to set the output pulse width of these AND circuits to more than $50 \mathrm{~ns}$ to eliminate the spurious coincidence background produced by phototube afterpulsing caused by light returning from the anode region to the photocathode. However, for this particular coincidence circuitry, it is important not to set the AND gates to lengths between 50 and 300 ns. Such a choice would permit accidental coincidences to occur within a scintillator and between scintillators, whereas real coincidences (which occur within 50 and $100 \mathrm{~ns}$ ) could occur only between scintillators. To prevent an imbalance in the circuitry that would yield negative assays, the AND gates are presently set to yield 400 -ns output pulse widths. These pulses are then clipped to $5 \mathrm{~ns}$ and ORed together by the LRS 365 AL dual fourfold logic unit.

Two autocorrelation circuits analyze these pulses (Fig. 4-5). The triple OP: that defines ime $=0$ for double coincioence events is the bottom half of the LRS dual fourfold logic unit referred to in the preceding paragraph. Additional LRS 622 coincidence units are then used to generate double coincidences (actually real plus accidental events) and accidental double coincidences. These data are sent to the CAMAC scalers, and the data analysis program computes the net real coincidences as the difference between the double and the accidental double coincidence response. To balance the circuit at high count rates, the outputs of the one-shots that define the coincidences are clipped to $38.4 \mathrm{~ns}$ and 38.8 ns for double and accidental double coincidences, respectively. The second autocorrelation circuit is used for triple coincidences. The output of the double coincidence module is sent to the upper half of the fourfold logic unit to define time $=0$ for triple events. Thus, the triple events are actually generated by looking for pairs of double events. For the triples circuit, count rates are so low that it is not necessary to define the lengths of the one-shots with clipping lines.

The above circuitry processes both neutrons and gamma rays that are detected by the plastic scintillators. The octal discriminator thresholds of $-100 \mathrm{mV}$ are equivalent to $95 \mathrm{keV}$ of electron energy deposition." This energy corresponds to $220-\mathrm{keV}$ gamma rays and $650-\mathrm{keV}$ neutrons. Although these thresholds imply that the AmLi neutron source $60-\mathrm{keV}$ gamma rays and $300-$ to $500-\mathrm{keV}$ neutrons will not be detected, it is possible for the neutrons to be detected by means of the $n+p \rightarrow d+$ gamma +2.2 $\mathrm{MeV}$ reaction. 


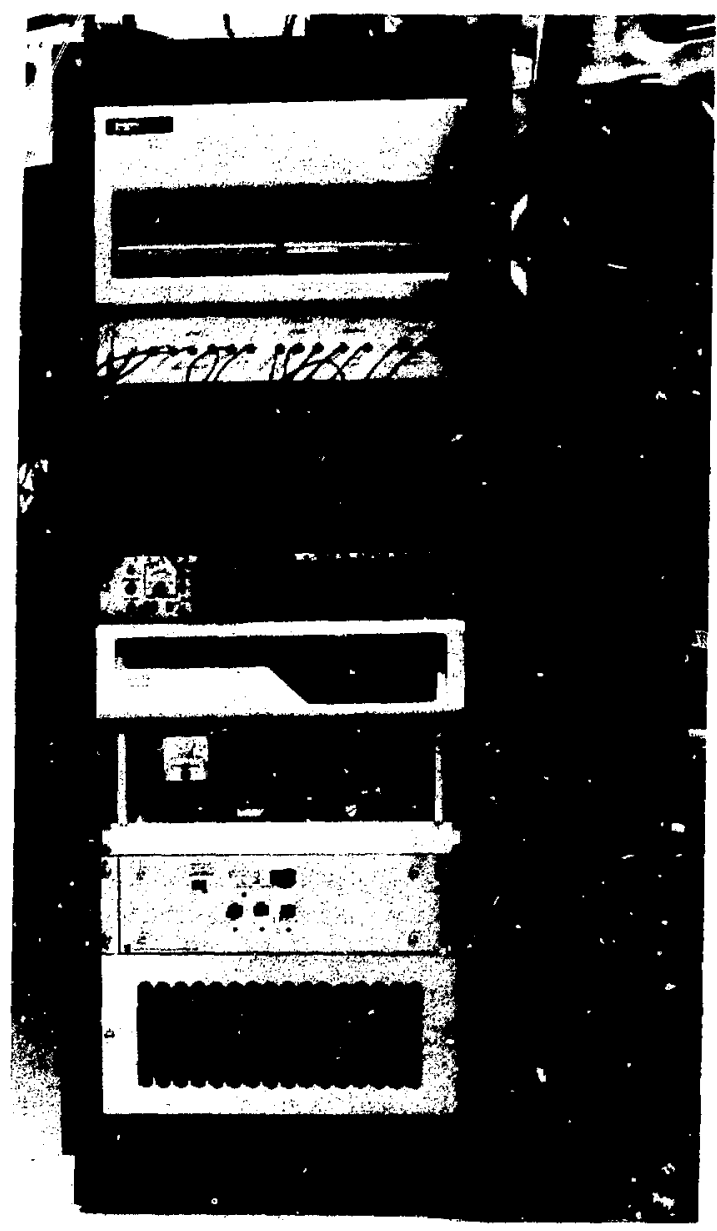

TABLE IV-2

CAMAC CKATE EQUIPMENT LIST

\begin{tabular}{cl} 
Address & \multicolumn{1}{c}{ Module } \\
\hline & \\
1,2 & LRS 612 12-channel PMT amplifier \\
3,4 & LRS 623 octal discriminator \\
5,6 & LRS 622 quad coincidence \\
7,8 & LRS 365 Al 4-fold logic unit \\
9,10 & LRS 622 quad coincidence \\
11,12 & Canberra 2015 amp/SCA \\
13,14 & Canberra 3105 HV power supply \\
15 & Unused \\
16 & ByRa 5601 TTL-NIM converter \\
17 & KS 3290 Dataway display \\
18 & LRS 2551 12-channel scaler \\
19,20 & KS 3552 ADC \\
21 & KS 3530 relay multiplexer \\
22,23 & Assay control module \\
24,25 & SEC DC-11 crate controller
\end{tabular}

Fig. 4-3.

Electronics cabinet used for FNC.

As illustrated in Fig. 4-6, (n,n), (n,gamma), and (gamma,gamma) coincidences a : 2 possible. The tıme difference between both events is a function of the size and shape of the detector cavity and the neutron energy spectrum. Figure 4-6 shows the approximate time difference spectrum present in the FNC with 5 $\mathrm{cm}$ of lead between the cavity and the scintillarors. ${ }^{4}$ (Gamma,gamma) events are largely rejected by the LRS $365 \mathrm{AL}$ fourfold logic unit, which has a pulse-pair resolution of $5 \mathrm{~ns}$. Because the gates (one-shots) are set to about 40 ns, the FNC accepts roughly $72 \%(n, n)$ and $28 \%$ (n,gamma) events in the time interval 5 to 45 ns (Fig. 4-6).

The following list of modifications to the SEC DC-11 crate controller were done by Atwell.* These crate controller modifications eliminate the problem of sending an unwanted DATIP CAMAC command on a computer MOV instruction. These changes are incorporated into later DC-11 models.

(1) Cut connectior. Letween pin 4 (H9) and pin 10 (E8). This disconnects NOREAD bit so it is not used.

(2) Connect pin 4 (H9) to pin I (H3). This connects SIFF and DMAOP output.

*T. L. Atwell, one of the authors of this report. 


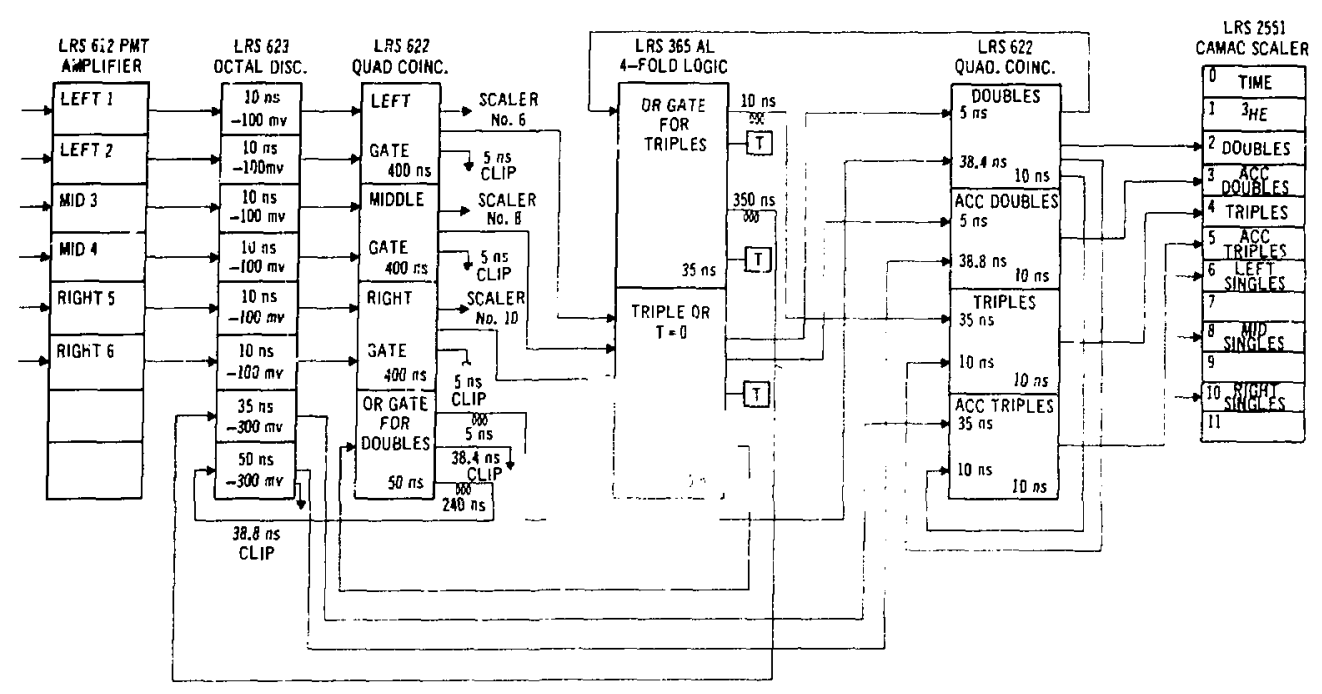

Fig. 4-4.

FNC fast-timing circuit diagram.

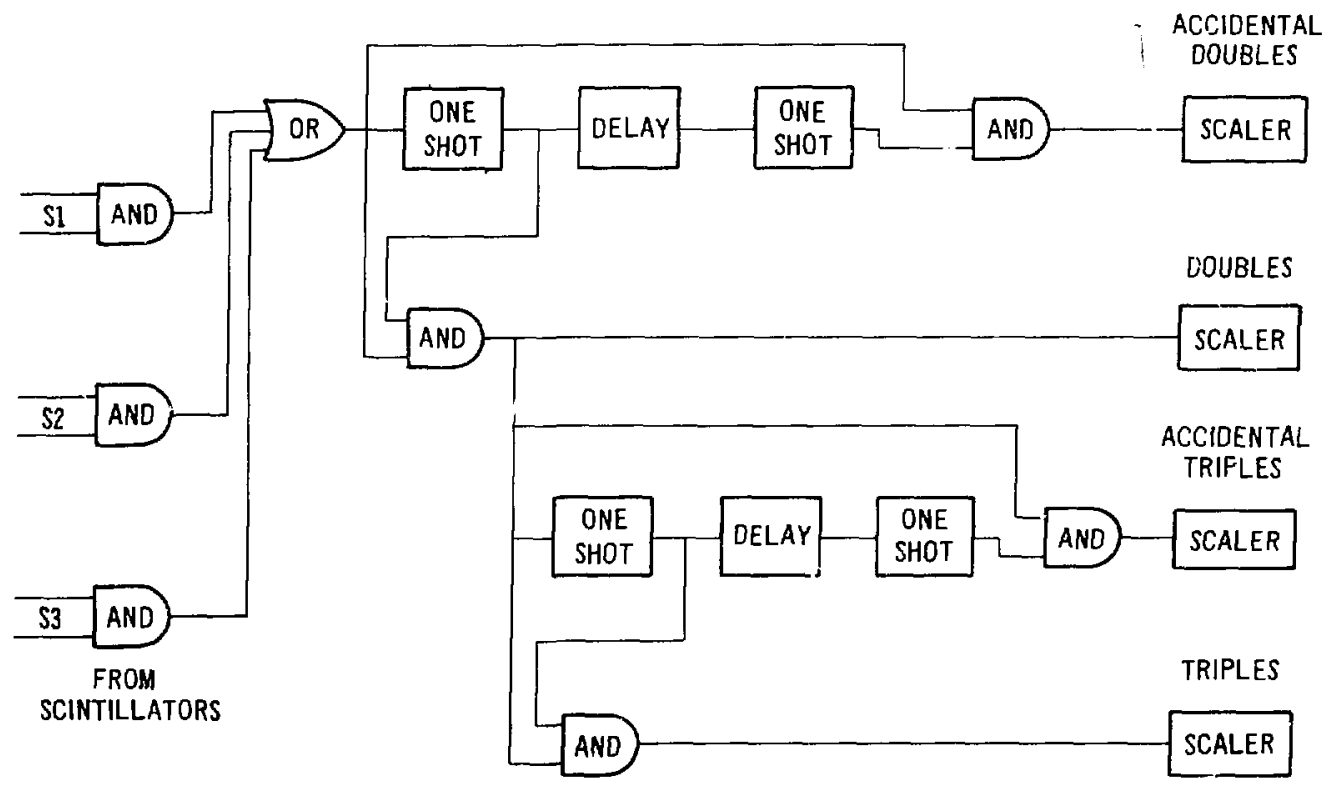

Fig. 4-5.

Autocorrelation circuits used to generate fast double and triple coincidences. 

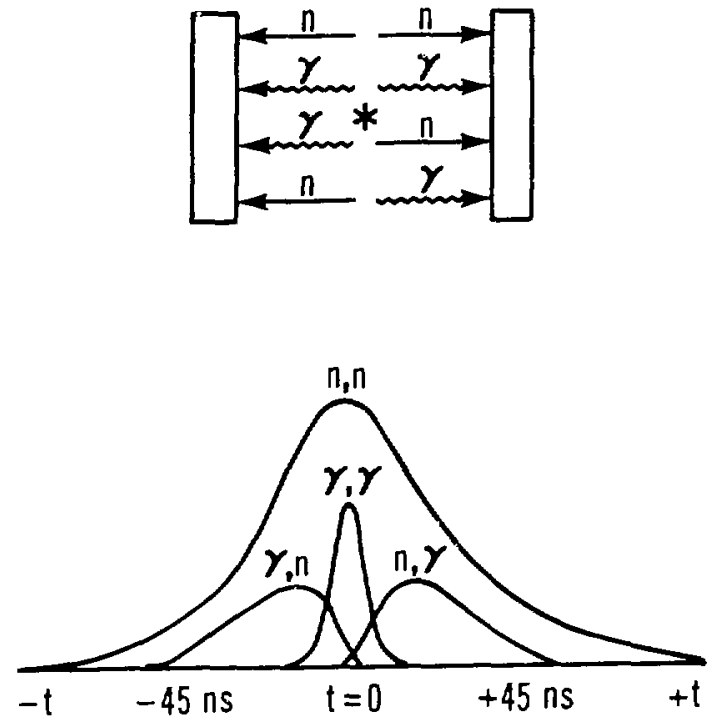

TIME DIFFERENCE
Fig. 4-6.

Idealized timing distributions for the FNC. The various possible coincidence distributions are drawn approximately to scale.

\section{CONTRIBUTIONS OF VARIOUS \\ COINCIDENCES FOR DIFFERENT \\ COINCIDENCE GATES}

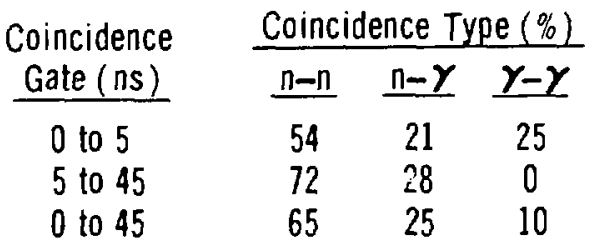

(3) Cut connection between pin $5(\mathrm{H} 9)$ and pin $4(\mathrm{G} 1)$. This disconnects inverter on $\overline{\mathrm{DATIP}}$.

(4) Connect pin 3 (G1) to pin 5 (H9). This connects DATIP to pin 5 (H9).

(5) Cut connection between pin 6 (H9) and pin 9 (G9). This disconnects the output of the NAND gate (H9) from BUSYFF.

(6) Tie pin 9 (G9) to $+5 \mathrm{Vdc}$ (input not used).

(7) Cut connection between pin $2(\mathrm{H} 4)$ and pin $12(\mathrm{H} 3)$. This disconnects inverter $(\mathrm{H} 4)$.

(8) Connect pin $12(\mathrm{H} 3)$ to pin $6(\mathrm{HP})$. This NANDS $\overline{\text { DATIP }}$ with SIFF and $\overline{\text { DMAOP }}$ to prevent HDR from clocking on DATIP cycles.

\section{Assay Control Module}

This module was designed and built by T. Atwell, B. Tippens, K. Henneke, K. Johnson, and T. Van Lyssel to interface assay control, temperature read-out, and source drive motor control to the computer. 
The module is a CAMAC module with slot address 22 . The custom circuitry is wire-wrapped on an SEC WW-04 double-width CAMAC prototype module. The prototype module has an 86-pin connector, which provides de power and all signals from the dataway. Circuitry is provided to decode CAMAC functions $(\mathrm{F})$, subaddresses $(\mathrm{A})$, slot address $(\mathrm{N})$, and all strobe timing and gating signals, busy, inhibit, look-at-me (LAM), and clear commands (S1, S2, C, Z, B, I, L, X, Q). A 36-pin rear panel connector is provided for external inputs or outputs. Table IV-3 lists the present allocation of pin locations on this connector, and Fig. 4-7 is a schematic of the custom wire-wrap circuitry on the module.

TABLE IV-3

ASSAY CONTROL MODULE REAR-PANEL CONNECTOR LAYOUT

Pin No. Function

thermistor No. 3 thermistor No. 1 thermistor No. 3 thermistor No. 1 thermistor No. 3 thermistor No. 1 thermistor No. 4 thermistor No. 2 thermistor No. 4 thermistor No. 2 thermistor No. 4 thermistor No. 2 temperature No. 1 data gnd temperature out temperature No. 2 data gnd temperacure out temperature No. 3 data gnd temperature out temperature No. 4 data gnd temperature out solid state relay - gnd solid state relay $+5 \mathrm{~V}$ in process LED (green) requested LED (yellow) request assay button ready LED (red) preamp power $+12 \mathrm{~V}$ brake relay ground $+5 \mathrm{~V}$

$$
\text { down relay }
$$$$
\text { up relay }
$$
down limit switch up limit switch signal $A$ signal $\mathbf{B}$
Cable

Destination

Pin No. green photomultiplier tubes

green

red

red

brown

brown

green

green

red

red

brown

brown

coax

$\operatorname{coax}$

coax

$\operatorname{coax}$

$\operatorname{coax}$

coax

$\operatorname{coax}$

$\operatorname{coax}$

black

brown

red

yellow

blue

green

coax center

blue-green

black

yellow-white

brown-red

white

black

blue-green

brown-red

top of cabinet

HV power supply

CAMAC crate

relay mutliplexer

relay mutliplexer

relay multiplexer

relay multiplexer

relay mutliplexer

relay multiplexer

relay multiplexer

relay multiplexer

request assay box

request assay box

request assay box

request assay box

request assay box

request assay box

unused

Minarik controller

encoder, limit switch

Minarik controller

Minarik controller

source holder

source holder

optical encoder

optical encoder
15

$15 \mathrm{R}$

14

$14 R$

13

13R

12

12R

A

B

C

D

E

$\mathbf{F}$

$\mathbf{E}$

B

request assay box, Minarik,encoder

D

B

D 


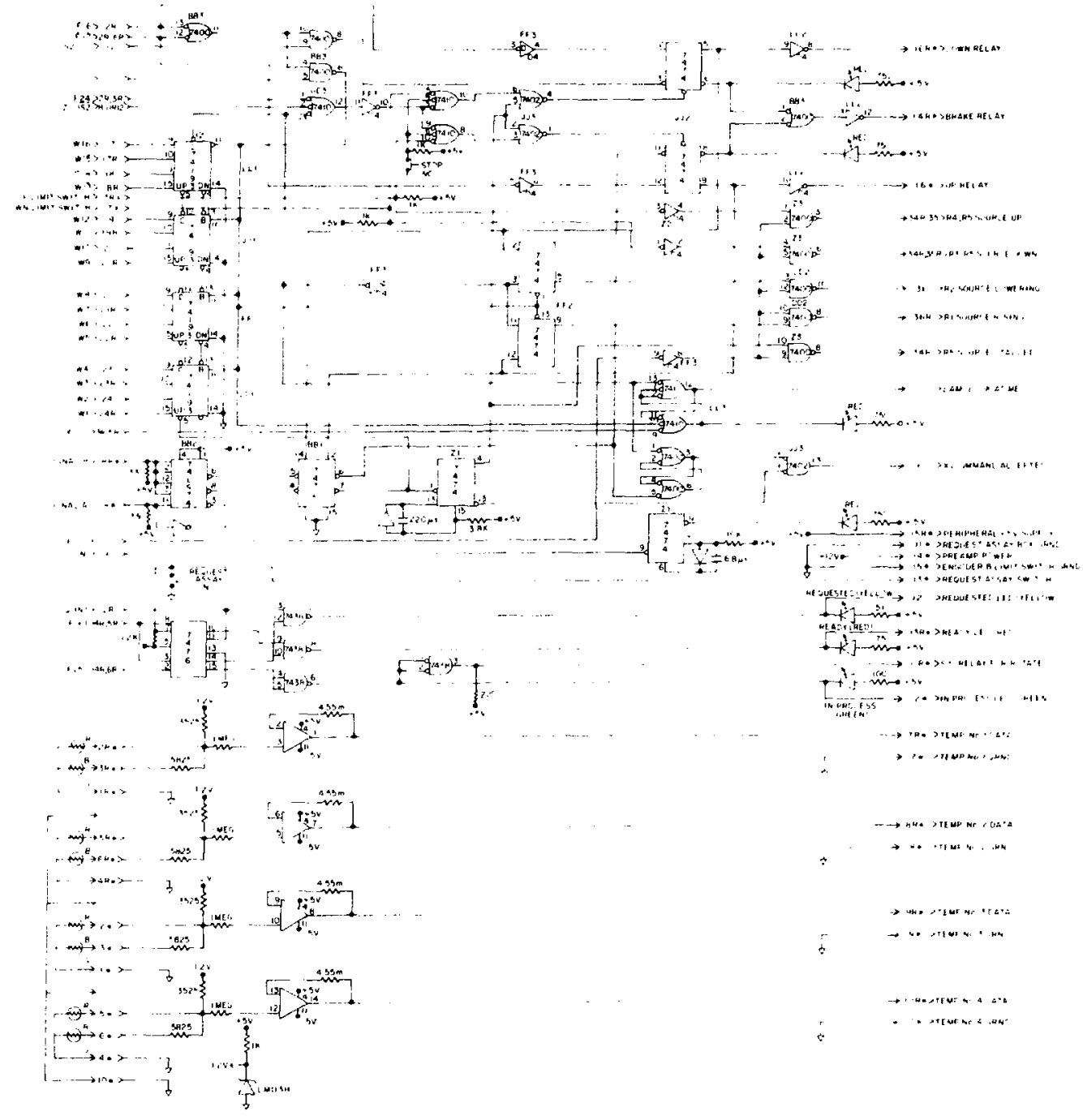

Fig. 4-7.

Assay control module wire-wrapped circuitry.

The assay control circuitry is shown in the center of Fig. 4-7. It consists of three light emitting diodes (LEDs) that indicate assay status: FNC ready for a newv assay (red), assay in process (green), new assay requested (yellow), and a request assay button. If the FNC is ready for a new assay (red LED on), pushing the request assay button will set the yellow LED and signal the computer that a new assay has been requested. The computer will have been waiting for the status variable $S$ to become $=0$; this is accomplished by returning $Q=1$ and $X=1$ from the assay control module. (For additional details on this sequence, refer to the DYMAC3 and the NAFD software listings.) The above assay control circuitry is replicated in the request assay box mounted underneath the glove box containing the FNC chassis, so the operator does not have to walk over to the electronics cabinet. The request assay box also contains an on-off switch for the sample holder rotator. If the rotator is not on, the request assay button on this box 
is disabled. The schematic oi this box is given in Fig. 4-8, and the electrical connections are included in Table IV-3. Neither request assay location has a button to stop an assay in progress. This is done using the software by typing CONTROL-C and GO TO 1000.

Four YSI 44211 thermistors, accurate to $\pm 1^{\circ} \mathrm{C}$ or better, are used to supply room, PMT, HV power supply, and CAMAC crate temperatures to the computer. With this information a temperature correction can be applied to the cbserved coincidence response. The thermistor signals are amplified by a factor of 4.55 by four LM 224 operational a mplifiers. These circuits are included in the assay control module and are shown on the lower portion of Fig. 4-7. The amplifier outputs are sent to the KS 3530 relay multiplexer and then converted to a digital signal by the KS 3552 optically isolated 12-bit analog-to-digital converter (ADC). The ADC is operated with a gain of one that covers a range of 0 to $5.12 \mathrm{~V}$.

The remaining circuitry in the assay control module is used to control the AmLi neutron source drive motor. This circuitry is shown in the upper half of Fig. 4-7. Information on the position of the source is obtained from up and down limit switches and from an optical rotation encoder mounted on the dc motor shaft used to drive the Teleflex cable. The encoder is a Trimstep model that supplies 50 pulses per revolution without a marker pulse. The pulses are complementary square waves obtained from TTL active pull-up. These pulses enter the circuit as signals $A$ and $\bar{B}$ and are used to increment $C 74193$ up-down counters. The computer uses the CAMAC write lines to set a specified number of pulses in these counters.

Source motion is initiated automatically by the computer by a CAMAC F(16) command (down) or an $F(17)$ command (up). These commands activate the down or up relays added to the modified Minarik motor controller (Fig. 4-9). A third relay has been added to this controller to act as a positive brake. The up and down relays are opened, and the brake relay is activated (1) when the up or down limit switch is set, (2) when the number of optical encoder pulses received by the up-down counters equals the number set on the write lines, (3) when encoder pulses are not being received but the up or down relay is activated, indicating that the source is stalled, or (4) when the manual stop button on the front panel of the assay control module is pushed. A computer request for source motion is indicated by a brief flash of the $\mathrm{N}$ (crate address) LED on the assay control module. When source motion is complete, the look-at-me (LAM) LED will come on until the computer responds to the module. The relay and limit switch connections between the assay control module and the modified Minarik motor controller are included in Table IV-3. Additional details on computer cortrol of the source drive are given in Sec. V (software).

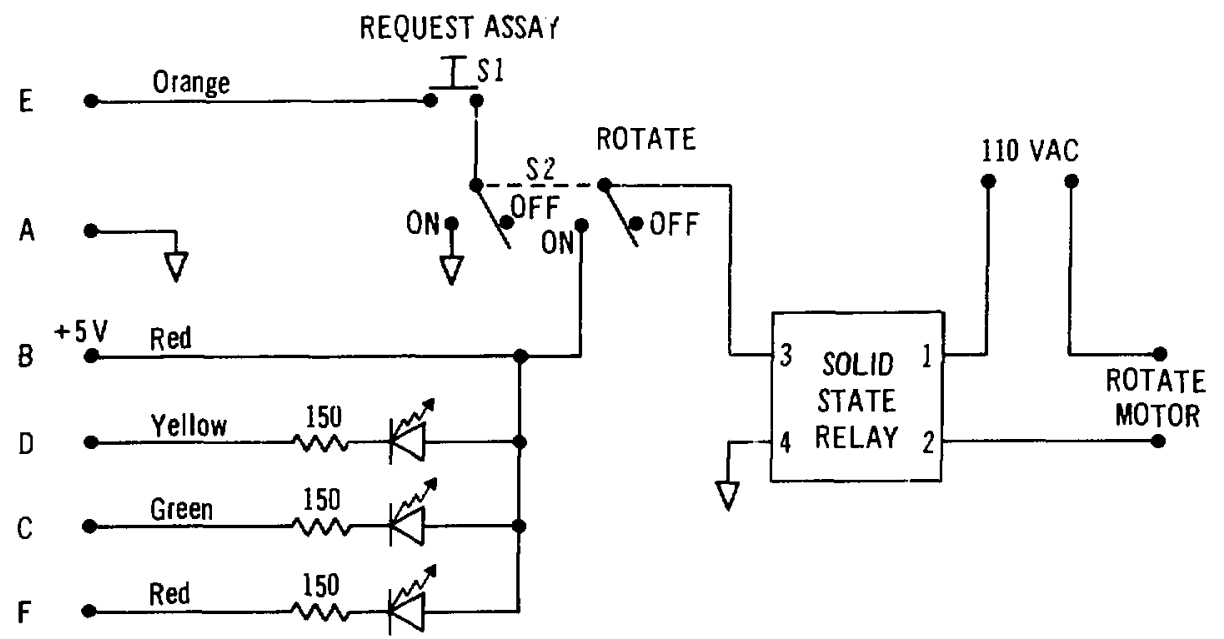

Fig. 4-8.

Request assay box schematic. 


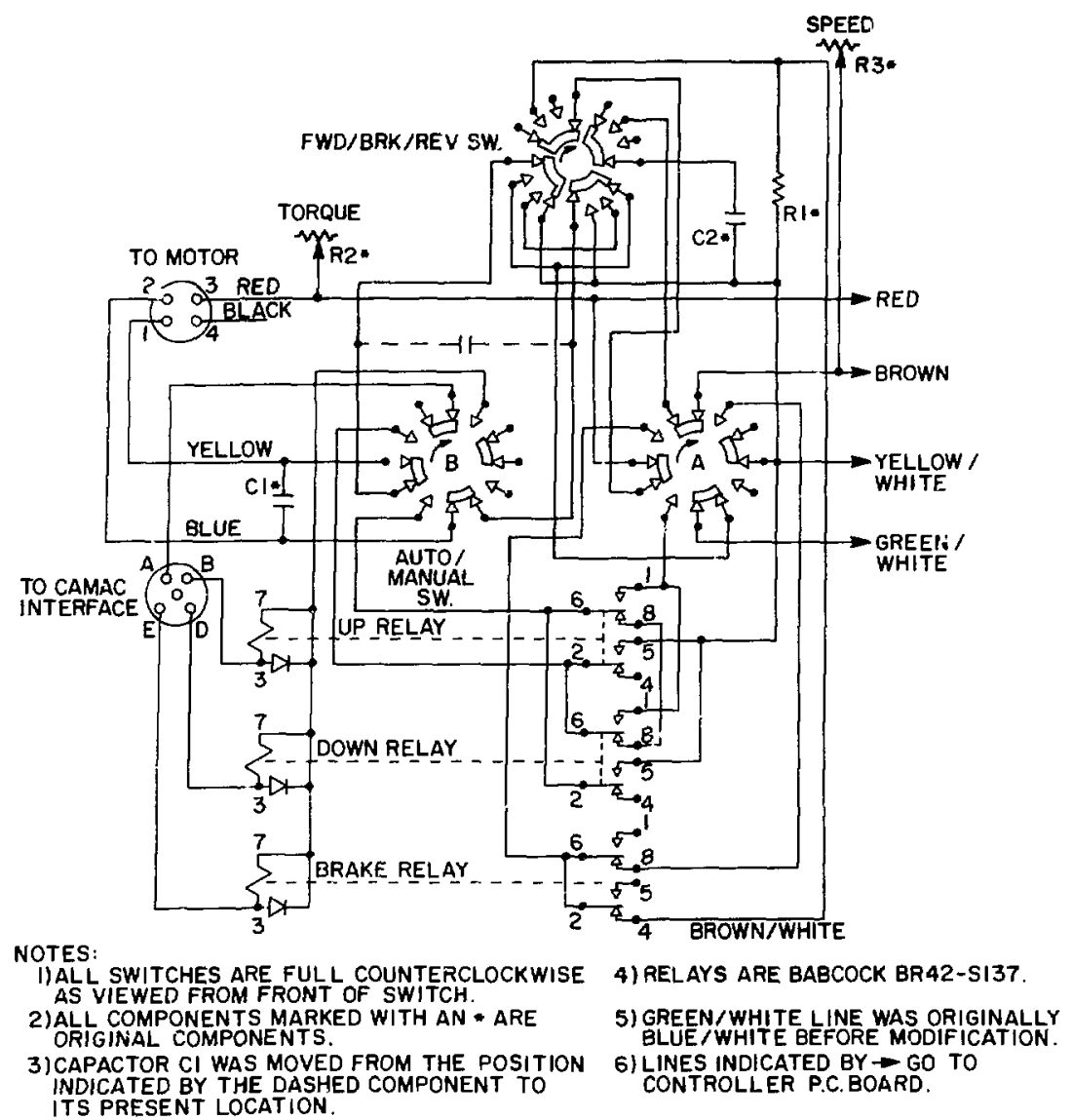

Fig. 4-9.

Modified Minarik motor controller schematic.

\section{Computer Configuration}

The FNC uses a DEC PDP 11/04-MC computer that requires $115 \mathrm{~V}(47-63 \mathrm{~Hz})$. The chassis is $5-\mathrm{i} / 4$ in. high and contains nine Hex (full-width) slots. Table IV-4 lists the boards mounted inside the chassis. The central processing unit (CPU) is ferrite core based, and the computer has $28-\mathrm{K}$ words of usable core memory. Core memory was selected for this computer so provision could be made in the future for recovery from a power failure in the production plant. An undesirable feature of this choice is that only 8 $\mathrm{A}$ of $+5 \mathrm{~V}$ are available for additional boards. Because of this limitation, a second terminal cannot be added to the computer although slot space is available for the additional serial line interface.

\section{SOFTWARE}

\section{A. Introduction}

The operating system for the DEC 11/04 computer is RT-11 Version 1. The programming language used up to the present time is Multi User-BASIC (MUBASIC). $\mathrm{R} T-\mathrm{I} I$ and MUBASIC use up a little 
TABLE IV-4

PDP-11/04-MC CONFIGURATION

\begin{tabular}{|c|c|c|c|}
\hline Component & Board Number & Description & $+5 \mathrm{~V} \mathrm{dc} A$ Required \\
\hline M7263 & M7263 & CPU & \\
\hline MM1 1-DP & & 16-K bytes core memory & \\
\hline MM1 1-DP & & 16-K bytes core memory & \\
\hline M9301-YB & M9301-YB & Bootstrap/emulator & \\
\hline KY11-LB & M7859 & Console register & 3 \\
\hline RX11-WB & M7846 & Dual floppy disk controller & 1.5 \\
\hline DL1 1-WB & M7856 & $\begin{array}{l}\text { Serial line interface and } \\
\text { line frequency clock }\end{array}$ & 2 \\
\hline \multirow[t]{2}{*}{ M9302 } & M9302 & Unibus terminator & \\
\hline & & & $6.5 \mathrm{~A}^{\mathrm{a}}$ \\
\hline
\end{tabular}

"Warning: This number must not exceed $8 \mathrm{~A}$.

more than half of the 32-K memory, leaving $14-15 \mathrm{~K}$ for programming. The FNC program is called DYMAC3 and uses about $7 \mathrm{~K}$ of memory. Thus additional computer memory is available, although, as mentioned in Sec. IV.G, the computer does not have enough de power for an additional terminal interface board.

A typical floppy disk should contain the following programs;

\begin{tabular}{|c|c|}
\hline MONITR.SYS & 46 19-OCT-77 \\
\hline DX.SYS & $219-$ OCT-77 \\
\hline TT.SYS & $219-$ OCT-77 \\
\hline LP.SYS & $219-\mathrm{OCT}-77$ \\
\hline PR.SYS & 2 19-OCT-77 \\
\hline PP.SYS & 2 19-OCT-77 \\
\hline SYSMAC.SML & $1819-$ OCT-77 \\
\hline MACRO.SAV & 31 19-OCT-77 \\
\hline LINK.SAV & 25 19-OCT-77 \\
\hline PIP.SAV & 14 19-OCT-77 \\
\hline EDIT.SAV & $1919-$ OCT-77 \\
\hline EXIT.BOO & 1 19-OCT-77 \\
\hline MUBASC.SAV & 66 19-OCT-77 \\
\hline INIT.BOO & 1 19-OCT-77 \\
\hline iŨR.DOO & 2 19-OCT-77 \\
\hline 2UNR.DOO & 2 19-OCT-77 \\
\hline BYE.BOO & $119-$ OCT-77 \\
\hline DIRECT.BAS & 6 19-ОСТ-77 \\
\hline RESEQ.BAS & 4 19-OCT-77 \\
\hline DELETE.BAS & 4 19-OCT-77 \\
\hline LSQFIT.BAS & 14 19-OCT-77 \\
\hline LSQDA I.DAS & 16 19-OCT-77 \\
\hline
\end{tabular}


LSQDA2.DAS

DYMAC3.BAS

RDINIT.DO1
16 19-OCT-77

$2516-\mathrm{NOV}-78$

$123-\mathrm{JUN}-78$

MONITR.SYS is the RT-1] system. MUBASC.SAV is the MUBASIC language. The $\mathrm{C}$ refers to CAMAC handlers written by T. L. Atwell that are described in Sec. IV. IUR.DOO is the one-user configuration file required by MUBASC.SAV. DIRECT.BAS, RESEQ.BAS, and LELETE.BAS are three BASIC programs written by T. L. Atwell to facilitate programming. DIRECT can be called to provide a floppy disk directory without exiting from MUBASIC. RESEQ can be used to resequence or renumber BASIC programs. DELETE can delete sections of coding from other programs. These three programs print self explanatory operating instructions when called. LSQFIT.BAS is the nonlinear least squares fitting routine used to generate calibration constants from the assay of known samples. LSQDA1.DAS and LSQDA2.DAS are data files used by this program. Section E describes LSQFIT. DYMAC3.BAS is the FNC operating program. Calibration constants and measurement control parameters are stored in RDINIT.DO1. Section IV B, C, and D describe DYMAC3.

\section{B. DYMAC3 Program Description}

This program is used to carry out passive or active-minus-passive assays. It handles communication with the operator, the floppy disk files, and the CAMAC crate: operation of the source-control motor; all data reduction and print-out; and some measurement control functions. The following assay decisions are made by the program.

1) The assay time is fixed at $500 \mathrm{~s}$ for passive measurement and $500 \mathrm{~s}$ for active measurement. Improved assay precision $c$ an be obtained by using the autocycle feature and averaging the results by hand.

2) After the operator selects the material type, the program decides whether an active measurement should be made (lines 1070-1125).

3) Oxide assays are usually passive but, if the singles/doubles ratio is above 70 (implying that tie oxide is impure), the program initiates an active assay (lines 2050-2060).

4) If the plutonium content of an unknown sample has been determined by both a passive and an active-minus-passive assay, the program chooses (within statistical errors) the smaller value as the final assay result (lines 3030-3050). Passive assays are inherently more precise but always overestimate the plutonium content if $(\alpha, n)$ reactions are present.

\section{Line-by-Line Program Description}

\begin{tabular}{|c|c|}
\hline $10-670$ & Initialization. \\
\hline 40 & $A=S Y S(6,80)$ sets line length to 80 characters. \\
\hline $110-200$ & These functions are described following line 6000 . \\
\hline $500-520$ & $\begin{array}{l}\text { If floppy disk is available, read stored parameters from VFl } \\
\text { into } V(100) .\end{array}$ \\
\hline $530-560$ & $\begin{array}{l}\text { Inspection or alteration of } \mathrm{V}(100) \text { using subroutine } 5200 \text {. This } \\
\text { is optional if floppy disk is available, mandatory if it is not. }\end{array}$ \\
\hline $570-600$ & $\begin{array}{l}\text { If elements of } V(100) \text { have been changed, these lines can be used } \\
\text { to replace the elements in VFl. }\end{array}$ \\
\hline $610-670$ & $\begin{array}{l}\text { Transfer stored parameters from } V(100) \text { to specific named } \\
\text { variables. }\end{array}$ \\
\hline
\end{tabular}


$1000 \quad$ Start assay (or sequence of assays) routine.

If initialization described above is still valid, program can be restarted by typing GO TO 1000.

1000 Go to subroutine 5400 and make sure source is down. When it is down print R.D. AVAILABLE

1020 Wait loop until request assay button is pushed.

1030-1040

$1060-1130$

$1150-1170$

1180-1200

$1210-1230$

Ask operator if sample is loaded and rotating. If so, go to subroutine 5500 and start passive assay. (Active assay is optional, but passive always comes first.) Operator now has about $8 \mathrm{~min}$ to answer the questions that follow. Answering $\langle C R>$ will skip to the next question. Answering $B$ will return to the preceding question.

Assay type (background, normalization) or material type (oxide, fluoride, electrorefined metal, oxide reduction metal, or residue) must be specified. Twelve is set accordingly.

If sample weight, diameter, and fill height are supplied, material and plutonium densities will be calculated.

If enrichment is supplied, an assay in total grams plutonium will be given. Otherwise, only ${ }^{240} \mathrm{Pu}$ (and maybe ${ }^{239} \mathrm{Pu}$ ) will be given. The variables E2 and E8 are available to calculate ${ }^{240} \mathrm{Pu}$ effective in the future. For the weapons-grade material assayed so far, effective ${ }^{240} \mathrm{Pu}$ is close to 1.02 times actual ${ }^{240} \mathrm{Pu}$, so this factor is being used.

Operator can enter NO to get one run, $\langle\mathrm{CR}>$ to get one run, or YES to get 100 runs; or he can enter a number to get a specific number of runs.

Go to Sec. 2000 , read temperature, and wait for passive assay to finish.

2000 If a sequence of runs is in process, start next p.ssive assay here. Go to subroutine 5400 and lower source, then go to subroutine 5500 and start passive assay.

2010 When passive assay is in progress, go to subroutine 5000 and cad temperature, then go to subroutine 5600 and wait for end of run.

2020 When run finishes, go to subroutine 4000 and do passive $(\mathrm{J}=0)$ data reduction.

2030 If the enrichment has been specified, convert assay to grams of total plutonium.

2040 For background, normalization, metal, and residues, go to active assay routine.

2050-2060 If the singles/doubles ratio is higher than that expected for clean oxide, go to active assay routine.

$3000 \quad$ Active assay routine.

3000 Go to subroutine 5400 and raise source. Go to subroutine 5500 and start assay.

3010 Go to subroutine 5000 and read temperature; then go to subroutine 5600 and wait for end of run.

3020 When run finishes, go to subroutine 4000 and do active $(\mathrm{J}=2)$ data reduction. 
If the enrichment has been specified, convert active assay to grams of total plutonium. Use the active assay as the final result only if the passive assay is larger than the active assay by more than 1 -std-dev.

3050 For electrorefined metal use passive assay. Active is done only to help calibration of reduction metal.

$3100-3120$ If the enrichment has been specified, and if the assay was not a background or normalization run, print the final result in grams of total plutonium. This final result may be based on the passive or on the active-passive assay.

and wait for new input.

$4000 \quad$ Active or passive data reduction routine. Determine length of run in seconds.

$4020-4060$

$4070-4100$ Read scalers. Transfer ${ }^{3} \mathrm{He}$ and singles data to named variables. If all singies data were 0 , repeat read-out up to 10 times if necessary. Print warning if read-out still 0 .

Print scaler data.

Evaluate real double and triple coincidence rates and total singles rates. Compute associated statistical errors.

Check that left, middle, and right scintillator counts are equal within $50 \%$. Print warning if not.

$4180-4200$ Calculate temperature correction for double coincidences. The actual correction for this driver has not been measured. The slope $\alpha=-0.00137 /{ }^{\circ} \mathrm{C}$ and the offset temperature $\mathrm{T}$ $=35.75^{\circ} \mathrm{C}$ were determined for the Oak Ridge National Laboratory random driver. The temperature correction is of the form

true rosponse $=\frac{\text { measured response }}{1+\alpha\left(\mathrm{T}-\mathrm{T}_{0}\right)}$.

T is now taken to be the mean of the crate, HV supply, and PMT temperatures. The error in the correction is based on an estimated $20 \%$ error in the determination of $a$. Do not apply correction to data, but print warning if calculated correction is outside range 1.00-1.03.

4210-4220 Subtract background (as determined during background update) from double and triple coincidences.

4230-4240 If this was an active assay, subtract the passive (background corrected) assay to get the net active-minus-passive assay.

4250-4300 Calculate the flux correction (active assay only). Subtract passive ${ }^{3} \mathrm{He}$ flux rate, then divide active-passive flux rate by reference rate determined during active background update. This is the flux ratio. Then

true response $=\frac{\text { measured response }}{1+0.217^{\star}(\text { flux ratio }-1)}$.

The coefficient 0.217 was determined in labora:ory tests before installation of the FNC in the plant. In practice a flux correction greater than $1 \%$ was never encountered. Thus the correction is not applied to the data, but a warning is printed if it ever exceeds $1 \%$. 
Calculate self-multiplication correction. This correction is based on triple coincidence/double coincidence ratios. Let $r=\frac{T_{\text {meas }} / D_{\text {meas }}}{T_{\text {ref }} / D_{\text {ref }}}=\frac{M(4)}{M(J)}$. $T_{\text {ref }} / D_{\text {ref }}$ was estimated at 0.007 for both actire and passive assays on the basis of data taken earlier with this instrument. Then subroutine 5300 is called to calculate the correction.

4350 Select appropriate set of calibration constants. Use oxide calibration for background, and normalization runs.

4360 Apply passive or active normalization (as determined during normalization update) to corrected double coincidence rate.

4370 Assay in grams of ${ }^{240} \mathrm{Pu}$ (passive assay) or ${ }^{239} \mathrm{Pu}$ (active-passive assay) is given by

mass $=\frac{A^{*} \text { response }}{1+B^{*} \text { response }}$.

To determine A and B, it is necessary to do the LSQFIT of salibration standaris in this form also, although in the case of calibration standards, it is really the response that is the dependent variable.

4375-4380 The error is given by the square root of

$$
\frac{A^{2} O R^{2}}{(1+B R)^{4}}+\frac{R^{2} O A^{2}}{(1+B R)^{2}}+\frac{A^{2} R^{4} O B^{2}}{(1+B R)^{4}}-\frac{2 A R^{3} \operatorname{cov}(A, B)}{(1+B R)^{3}} \text {, }
$$

where $\mathbf{R}$ is the corrected, normalized double coincidence response.

$4390-4460$

Compute material density if sample weight, diameter, and fill height were supplied. Compute plutonium density if enrichment was supplied. For metals and residues, sample diameter and fill height are assumed known. Print results of preceding data reduction routine. Prepare for background or normalization update if this assay was I BKG or NOR run. Open floppy disk file if disk is available.

Background update routine. Check AmLi source strength on active background run. Store measured backgrounds and ${ }^{3} \mathrm{He}$ flux rate. Update floppy disk if it is available.

4800-4890 Normalization update routine. Use passive corrected coincidence rate obtained with normalization standard to set both passive and active normalization if response has changed by more than 2 std dev. Currently standard is STD-9, corrected coincidence response $=\mathbf{2 3 2}$ counts $/ \mathrm{s}$. If the normalization is more than $10 \%$ away from one, raise or lower the high voltage as needed and repeat calibration. Update the floppy disk if it is available. 
$5000-5060$

$5100-5150$

$5200-5220$

$5315-5320$

5325

5330.5345

$5350-5355$

$5360-5365$

$5375-5380$
Subroutine to read temperature. The conversion from ADC reading (X9) through temporary variable $V(\mathrm{k})$, to temperature $\mathrm{U} 1,2,3,4$ is done using T. Van Lyssel's* calibration data of 3/31/78.

Subroutine to read ADC for temperature. Wait $1 \mathrm{~s}$, start ADC conversion, and read value into X9. Do this 11 times, and repeat if difference between highest and lowest reading is more than 20. If not, discard highest and lowest reading, and return the average of the other nine as the $\mathrm{ADC}$ result (X9). Subroutine to check or change contents of VFI(100) $=$ RDIT.DO1, as represented by V(100). As each variable is typed out, the operator can enter a new value, enter $B$ to backspace, or leave the current value unaltered by typing $\langle\mathrm{CR}\rangle$ or $\mathrm{O}\langle\mathrm{CR}\rangle$. Self-multiplicatiun correction subroutine. Select value of $\alpha=$ numbir of $(\alpha, n)$ neutrons/number of spontaneous-fission neutrons appropriate for each material category; $\alpha=21$ for $\mathrm{PuF}_{4}$ was an empirical choice that is no longer correct.

Set correction ractor $=1$ for background runs.

Set correction factor $=1.217$ for (STD-9) normalization runs. Solve a quadratic for the net isakage multiplication $\mathrm{M}$

$[4.709+3.735 \alpha] \mathrm{M}^{2}-[0.369 \mathrm{r}+6.510+4.9800] \mathrm{M}$

$+[0.17 r+2+1.245 \alpha]=0$.

If this value of $M$ is within 2 std dev of unity, set $M=1$. The statistical erro in $M$ is given by an approximate formula.

The self-multiplication correction factor is

$M(8)=0.8547 M(2.17 M-1)+1.282 a(M-1)(3 M-1)$.

The statistical error in $M(8)$ is again given by an approximate formula.

For active assays of the normalization standard, the self-multiplication correction factor is $1.117 . \mathrm{M}$ is the same as before.

For other active assays, the net leakage multiplication $\mathbf{M}$ derived during the passive assay is again used. But the equation for the correction factor is modified by replacing the spontaneous-fission multiplicity $v_{\mathrm{S}}=2.17$ by an induced fission multiplicity $v_{\mathrm{I}}=3$ and dropping the $(\alpha, n)$ term. The result is

$M(8)=0.5(3 M-1)(M)$.

The above equations are approximate equations that were used to apply self-multiplication corrections to the FNC data

*T. Van Lyssel, one of the authors of this report. 
during the test and evaluation (1978-1979) of the instrument. The equation for $M$ is based on a derivation similar to one previously published. ${ }^{5}$ The correction factor is

$\frac{M v_{S}\left(M v_{S}-1\right)(1+\beta)}{v_{S}\left(\nu_{S}-1\right)}$,

where $\beta$ is defined in the above paper.

A better correction factor, which is based on a summation of fission chains through all orders, is now available for passive assays. It should be incorporated into the software for any future assay campaigns. A derivation of the new correction factor has not yet been published. However, the correction is available in the following BASIC routine.

let $R 2=r=M(4) / M(J)$

let $\mathrm{Al}=\alpha=\mathrm{L}(\mathrm{I} 2)$

$64 \mathrm{P}=-(1.738+2.128 * \mathrm{~A} 1+.140135 * \mathrm{R} 2+.140135 * \mathrm{R} 2 * \mathrm{~A} 1) /(1+\mathrm{A} 1)$

$66 \mathrm{Q}=\left(.914534+1.38^{*} \mathrm{~A} 1+.141163^{*} \mathrm{R} 2+.195816^{*} \mathrm{R} 2 * \mathrm{~A} 1\right) /(1+\mathrm{A} \mathrm{I})$

$68 \mathrm{~S}=-\left(.135948+.252^{*} \mathrm{~A} 1+.041614^{*} \mathrm{R} 4+.055681^{*} \mathrm{R} 2 * \mathrm{~A} 1\right) /(1+\mathrm{A} 1)$

$70 \mathrm{~A}=\mathrm{Q}-\mathrm{P} * \mathrm{P} / 3$

$72 \mathrm{~B}=.074074 * \mathrm{~F}^{3}-\mathrm{P}^{*} \mathrm{Q} / 3+\mathrm{S}$

$74 \mathrm{~W}=\mathrm{B}^{2} / 4+\mathrm{A}^{3} / 27 \quad$ IF $\mathrm{W}<\mathrm{O}$ GO TO 90

$76 \mathrm{~A}=(-\mathrm{B} / 2+\mathrm{SQR}(\mathrm{W}))^{(1 / 3)} \mathrm{B}=(-\mathrm{B} / 2-\mathrm{SQR}(\mathrm{W}))^{(1 / 3)}$

$78 \mathrm{X}=\mathrm{A}+\mathrm{B} \quad$ GO TO 110

$90 \mathrm{~T}=\operatorname{SQR}\left(-4^{*} \mathrm{~A}^{3} /\left(27^{*} \mathrm{~B}^{2}\right)-1\right) \quad \mathrm{F}=\mathrm{ATN}(\mathrm{T})$

$100 \mathrm{X}=2^{*} \mathrm{SQR}(-\mathrm{A} / 3)^{*} \mathrm{COS}(\mathrm{F} / 3)$

$110 \mathrm{M}=\mathrm{X}-\mathrm{P} / 3$

$115 \mathrm{Cl}=3.4528^{*}(\mathrm{I}+\mathrm{A} 1)^{*} \mathrm{M}^{2}-\left(3.4781+4.8247^{*} \mathrm{~A} 1\right)^{*} \mathrm{M}+1.0253+1.3719^{*} \mathrm{~A} I$

$\mathbf{M}=$ net leakage multiplication $=\mathbf{M}(6)$.

$\mathrm{Cl}=$ self-multiplication correction factor $=\mathrm{M}(\mathrm{R})$.

For active assays a similar correction could be derived, but this has not been done yet.

$5400 \quad$ Subroutine to check or change AmLi source position.

5410 Initial number of steps to motor will be 410 , which should be the full length of travel required from one microswitch to the other. Total number of steps traveled so far $=0$.

5420 Check status of microswitches.

5430 If the source is at the upper limit, and if this is the desired position, return.

5440 If the source is at the lower limit, and if this is the desired position, return.

5450 If the source is stalled, announce this and proceed.

5460 If the motor has been asked to move more than 424 steps by this time (and the proper limit switch has still not been set), give up.

5470 If the source has already been moved (and the proper limit switch has not been set), set the number of steps for the next command to the motor at 5 .

5480 Send command to motor, wait for source to move, increment record $-?$ total travel, and return to start of subroutine to check limit switches. 
$5500 \quad$ Subroutine to start assay.

$5510 \quad$ Set crate inhibit, no read bit.

$5530 \quad$ Clear scalers.

5540 Remove inhibit (this starts scalers). Start timei routine for 30000 cycles or $500 \mathrm{~s}$. To actually measure the time interval, save the starting time in ZO\$.

$5550 \quad$ Turn on assay in prcess light.

$5600 \quad$ Subroutine to stop assay.

5600 Wait for timer function to time out (X8 goes to +1 from -1$)$. Set crate inhibit (this stops scalers). Record stopping time in Z1\$.

$5610 \quad$ Turn off assay in process light, turn on ready light.

C. DYMAC3 Variable List

\begin{tabular}{|c|c|c|c|}
\hline $\mathbf{A}(0)$ & passive daily normalization & $\mathrm{D}(3)$ & error \\
\hline$A(1)$ & error & $D(4)$ & passive net dbls, bkg corr. \\
\hline$A(2)$ & active daily normalization & $\mathrm{D}(5)$ & error \\
\hline$A(3)$ & error & $\mathrm{D}(6)$ & active net dbls, bkg corr. \\
\hline$A(4)$ & & $D(7)$ & error \\
\hline$A(5)$ & & $\mathrm{D}(8)$ & passive/active corrected doubles \\
\hline$A(6)$ & & $D(9)$ & error \\
\hline$A(7)$ & & $\mathrm{D}$ & sample diameter \\
\hline$B(0)$ & passive net doubles bkg & D\$ & flag for floppy disk availability \\
\hline$B(1)$ & error & $\mathrm{E}$ & effective ${ }^{200} \mathrm{Pu}$ enrichment \\
\hline $\mathrm{B}(2)$ & active net doubles bkg & E0 & per cent ${ }^{240} \mathrm{Pu}$ \\
\hline$B(3)$ & error & E2 & per cent ${ }^{242} \mathrm{~Pa}$ \\
\hline $\mathbf{B}(4)$ & passive net triples bkg & E8 & per cent ${ }^{238 \mathrm{pu}}$ \\
\hline$B(5)$ & error & E\$ & $+/-$ symbol \\
\hline$B(6)$ & active net triples bkg & Fo & temperature correction \\
\hline $\mathrm{B}(7)$ & error & F1 & error \\
\hline$B(8)$ & passive totals bkg & $G$ & ADC gain $1=5.12$ V F.S. \\
\hline $\mathrm{B}(9)$ & error & & $2=512 \mathrm{mV}$ F.S. \\
\hline $\mathrm{B}(10)$ & active totals bkg & & $3=51.2 \mathrm{mV} \mathrm{F.S.}$ \\
\hline$B(11)$ & error & $\mathrm{H}$ & sample fill height \\
\hline$C(I, 0)$ & passive calib $\mathrm{A}$ & $\mathbf{I}$ & index \\
\hline$C(I, I)$ & error $6 \mathrm{~A}$ & $\mathrm{I} 2$ & sample type $1=$ oxd $4=$ PRP \\
\hline$C(I, 2)$ & active calib $\mathbf{A}$ & & $2=$ flu $5=$ res \\
\hline $\mathrm{C}(\mathrm{I}, 3)$ & error $6 \mathrm{~A}$ & & $3=$ PMP $12=$ norm. \\
\hline $\mathrm{C}(\mathrm{I}, 4)$ & passive $B$ & $\mathbf{J}$ & assay type $0=$ passive \\
\hline$C(I, 5)$ & error & & $2=$ active \\
\hline$C(I, 6)$ & active $B$ & $\mathbf{K}$ & index \\
\hline$C(I, 7)$ & error & $\mathrm{K}(0)$ & passive ${ }^{3} \mathrm{He}$ flux rate \\
\hline$C(I, 8)$ & passive covariance & $\mathbf{K}(1)$ & error \\
\hline$C(I, 9)$ & active covariance & $\mathrm{K}(2)$ & active ${ }^{3} \mathrm{He}$ flux rate \\
\hline \multirow[t]{5}{*}{$\mathrm{D}(0)$} & passive/active net doubles, & $\mathbf{K}(3)$ & error \\
\hline & uncorrected & $K(4)$ & passive bkg reference flix rate \\
\hline & error & $\mathbf{K}(5)$ & etror \\
\hline & passive/active temp. corr. dbls & $\mathrm{K}(6)$ & active bkg reference flux rate \\
\hline & & $\mathbf{K}(7)$ & error \\
\hline
\end{tabular}


$\mathrm{K}(8)$ passive/active flux ratio

$K(9)$ error

$\mathrm{K}(10)$ passive/active flux correction

K(11) error

L(12) alpha $=\mathbf{N}_{\alpha \mathfrak{n}} / \mathbf{N}_{\mathrm{SF}}$

L2 temporary variable for quad. formula

L3 temporary variable for quad. formula

L4 temporary variable for quad. formula

M(0) passive triples/doubles reference

M(1) error

M(2) active triples/doubles reference

M(3) error

M(4) passive/active measured triples/doubles

M(5) error

M(6) passive/active leakage mult.

M(7) error

M(8) passive/active self-mult. corr. factor

$\mathrm{M}(9)$ error

$\mathrm{N}$ current or available run number

No total runs in cycle

N1 assay type $0=$ passive only

$$
\begin{aligned}
& 1=\text { active } O K \\
& 2=\text { active required }
\end{aligned}
$$

N2 not used

N3 source position $0=$ down desired

$$
1=\text { up desired }
$$

N4 limit switch status $16=$ stall

$$
\begin{aligned}
& 20=\text { down } \\
& 24=\text { up }
\end{aligned}
$$

N5 number of steps sent to motor

N6 total number of steps travelled

0

0\% one blank space

P1 material density

P2 plutonium density

S CAMAC status word

$S(0)$ time scaler/passive singles

S(1) ${ }^{3} \mathrm{He}$ scaler/error

$\mathrm{S}(2)$ dbl. coinc. scaler/active singles

$S(3) \quad$ acc. dbls. scaler/error

S(4) trip. coinc. scaler/active-passive sg.

$S(5) \quad$ acc. trip. scaler error

$\mathrm{S}(6) \quad$ left singles scaler

S(7) left singles overflow

S(8) middle singles scaler
S(9) middle singles overflow

$S(10)$ right singles scaler

S(11) right singles overflow

$\mathrm{T}$ count time

T(0) passive/astive net triples, uncorrected

$T(1)$ error

T(2) passive/active temp. corr. triples

$T(3)$ error

T(4) passive net triples, bkdg. corr.

$\mathrm{T}(5)$ error

T(6) active net triples, bkg. corr.

$T(7)$ error

$T(8) \quad$ passive/active corrected triples

$T(9)$ error

U1 room temperature

U2 crate temperature

U3 PMT temperature

U4 HV supply temperature

$\mathrm{V}$ sample volume

$V(100)$ virtual file temporary storage

W sample weight

$\mathrm{X}$ temporary variable

$\mathrm{X}(0)$ passive left totals

$X(1)$ error

$\mathrm{X}(2)$ active left totals

$\mathbf{X}(3)$ error

$X(4)$ active-passive left totals

$X(5)$ error

$X 8$ run timer

$X 9 \quad$ ADC reading

$Y$ temporary variable

$\mathrm{Y}(0)$ passive middle totals

$Y(1)$ error

$Y(2)$ active middle totals

$\mathrm{Y}(3)$ error

$Y(4)$ active-passive middle totals

$Y(5)$ error

Z1 ADC lowest reading

Z2 ADC highest reading

$Z(0)$ passive right totals

$Z(1)$ error

$Z(2)$ active right totals

$Z(3)$ error

$Z$ (4) active-passive right totals

$Z(5)$ error

Z\$ time string variable 
D. Calibration Constants for DYMAC3, as Stored in File RDINIT.DO1

\begin{tabular}{|c|c|c|c|c|}
\hline VF11 & & variable & meaning & value \\
\hline 1 & $C(1,0)$ & oxide & passive A & $0.1775(11 / 16 / 78)$ \\
\hline 2 & $C(1,1)$ & & error & 0.0015 \\
\hline 3 & $C(1,2)$ & & active $\mathrm{A}$ & 9.87 \\
\hline 4 & $C(1,3)$ & & error & 0.11 \\
\hline 5 & $C(1,4)$ & & passive $B$ & $8.4 \times 10^{-5}$ \\
\hline 6 & $C(1,5)$ & & error & $2.2 \times 10^{-5}$ \\
\hline 7 & $C(1,6)$ & & active $B$ & $-3.05 \times 10^{-3}$ \\
\hline 8 & $\mathrm{C}(1,7)$ & & error & $2.3 \times 10^{-4}$ \\
\hline 9 & $\mathrm{C}(1,8)$ & & passive covariance & $3.0 \times 10^{-8}$ \\
\hline 10 & $C(1,9)$ & & active covariance & $2.25 \times 10^{-5}$ \\
\hline 11 & $C(2,0)$ & fluoride & passive $A$ & $0.0738(10 / 4 / 78)$ \\
\hline 12 & $C(2,1)$ & & error & 0.0007 \\
\hline 13 & $C(2,2)$ & & active $\mathrm{A}$ & 0 \\
\hline 14 & $C(2,3)$ & & error & 0 \\
\hline 15 & $C(2,4)$ & & passive $B$ & $4.1 \times 10^{-3}$ \\
\hline 16 & $\mathrm{C}(2,5)$ & & error & $1.3 \times 10^{-4}$ \\
\hline 17 & $C(2,6)$ & & active $B$ & 0 \\
\hline 18 & $\mathrm{C}(2,7)$ & & error & 0 \\
\hline 19 & $C(2,8)$ & & passive covariance & $7.8 \times 10^{-8}$ \\
\hline 20 & $C(2,9)$ & & active covariance & 0 \\
\hline 21 & $C(3,0)$ & PMP & passive $A$ & $0.1812(1 / 3 / 79)$ \\
\hline 22 & $\mathrm{C}(3,1)$ & & error & 0.0012 \\
\hline 23 & $C(3,2)$ & & active $A$ & 14.14 \\
\hline 24 & $C(3,3)$ & & error & 0.16 \\
\hline 25 & $\mathrm{C}(3,4)$ & & passive $\mathbf{B}$ & $1.82 \times 10^{-5}$ \\
\hline 26 & $\mathrm{C}(3,5)$ & & error & $1.20 \times 10^{-5}$ \\
\hline 27 & $C(3,6)$ & & active $B$ & $-1.63 \times 10^{-3}$ \\
\hline 28 & $C(3,7)$ & & error & $9.4 \times 10^{-5}$ \\
\hline 29 & $C(3,8)$ & & passive covariance & $1.67 \times 10^{-B}$ \\
\hline 30 & $C(3,9)$ & & active covariance & $1.36 \times 10^{-5}$ \\
\hline 31 & $C(4,0)$ & PRP & passive $A$ & $0.1812(1 / 3 / 79)$ \\
\hline 32 & $C(4,1)$ & & error & 0.0012 \\
\hline 33 & $C(4,2)$ & & active $A$ & 14.14 \\
\hline 34 & $C(4,3)$ & & error & 0.16 \\
\hline 35 & $C(4,4)$ & & passive $B$ & $1.82 \times 10^{-5}$ \\
\hline 36 & $\mathrm{C}(4,5)$ & & error & $1.20 \times 10^{-5}$ \\
\hline 37 & $C(4,6)$ & & active $B$ & $-1.63 \times 10^{-3}$ \\
\hline 38 & $C(4,7)$ & & error & $9.4 \times 10^{-5}$ \\
\hline 39 & $C(4,8)$ & & passive covariance & $1.67 \times 10^{-8}$ \\
\hline 40 & $C(4,9)$ & & active covariance & $1.36 \times 10^{-5}$ \\
\hline 41 & $C(5,0)$ & residue & passive $A$ & $0.1775(11 / 16 / 78)$ \\
\hline 42 & $C(5,1)$ & (same as oxide) & error & 0.0015 \\
\hline 43 & $C(5,2)$ & & active $\mathbf{A}$ & 9.87 \\
\hline 44 & $\mathrm{C}(5,3)$ & & srror & 0.11 \\
\hline 45 & $C(5,4)$ & & passive $B$ & $8.4 \times 10^{-5}$ \\
\hline
\end{tabular}




\begin{tabular}{|c|c|c|c|}
\hline 46 & $C(5,5)$ & error & $2.2 \times 10^{-5}$ \\
\hline 47 & $C(5,6)$ & active $\mathbf{B}$ & $-3.05 \times 10^{-3}$ \\
\hline 48 & $C(5,7)$ & error & $2.3 \times 10^{-4}$ \\
\hline 49 & $C(5,8)$ & passive covariance & $3.0 \times 10^{-8}$ \\
\hline 50 & $C(5,9)$ & active covariance & $2.25 \times 10^{-5}$ \\
\hline 51 & $C(6,0)$ & passive $A$ & \\
\hline 52 & $C(6,1)$ & error & \\
\hline 53 & $C(6,2)$ & active $A$ & \\
\hline 54 & $C(6,3)$ & error & \\
\hline 55 & $C(6,4)$ & passive $B$ & \\
\hline 56 & $C(6,5)$ & error & \\
\hline 57 & $C(6,6)$ & active $B$ & \\
\hline 58 & $C(6,7)$ & error & \\
\hline 59 & $C(6,8)$ & passive covariance & \\
\hline 60 & $C(6,9)$ & active covariance & \\
\hline 61 & $A(0)$ & passive daily normalization & \\
\hline 62 & $A(1)$ & error & \\
\hline 63 & $A(2)$ & active daily normalization & \\
\hline 64 & $A(3)$ & error & \\
\hline 65 & $\mathrm{~B}(0)$ & passive net doubles bkg & \\
\hline 66 & $\mathrm{~B}(1)$ & error & \\
\hline 67 & $\mathrm{~B}(2)$ & active net doubles bkg & \\
\hline 68 & $\mathrm{~B}(3)$ & error & \\
\hline 69 & $B(4)$ & passive net triples bkg & \\
\hline 70 & $\mathrm{~B}(5)$ & error & \\
\hline 71 & $B(6)$ & active net triples bkg & \\
\hline 72 & $\mathrm{~B}(7)$ & error & \\
\hline 73 & $\mathrm{~B}(8)$ & passive totals bkg & \\
\hline 74 & $\mathrm{~B}(9)$ & error & \\
\hline 75 & $\mathrm{~B}(10)$ & active totals bkg & \\
\hline 76 & $\mathrm{~B}(11)$ & error & \\
\hline 77 & & reference temp $T_{0}$ & \\
\hline 78 & & reference temp slope $\alpha$ & \\
\hline 79 & $\mathrm{~K}(4)$ & flux rate during passive bkg & \\
\hline 80 & $\mathrm{~K}(5)$ & error & \\
\hline 81 & $\mathrm{~K}(6)$ & flux rate during active bkg & \\
\hline 82 & $\mathrm{~K}(7)$ & error & \\
\hline 83 & $\mathrm{M}(0)$ & trps/dbls passive reference & 0.007 \\
\hline 84 & $\mathrm{M}(1)$ & error & 0 \\
\hline 85 & $\mathbf{M}(2)$ & trps/dbls active raference & 0.007 \\
\hline 86 & $\mathbf{M}(3)$ & error & 0 \\
\hline 87 & $\mathbf{N}$ & next run number to be used & \\
\hline
\end{tabular}




\section{E. Documentation for Least Squares Fit Program (LSQFIT)}

This program is used to fit a set of standard cans run in the FNC to the functions

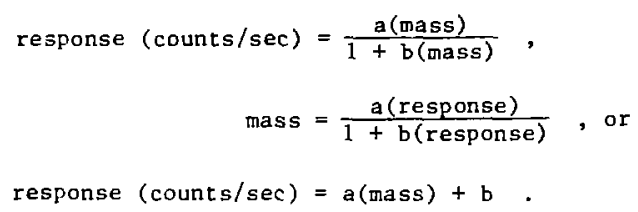

Because the first tw'o functions are nonlinear, the fit is carried out by iteration. For reasons of program compactness the last function is also fit by the same iterative routine, although an exact solution is possible in this case.

Lines 520 to 660 are used to initialize the program and open the files containing the values and names of the data points. These files are LSQDA1.DAS and LSQDA2.DAS for VF1 and VF28.

The program then prints its name and the list of allowed commands. The statements immediately below this list of commands will cause the program to branch to the desired subroutine and should be referred to by the reader to help locate the routines described below.

The command L-LIST DATA SET should be invoked first to look at the two data files that have just been opened (one for name strings and one for data points). For the purposes of this program the 2 data files are mixed together, but the combined file is treated as 10 separate data sets (1-10) that may contain up to 50 points each. The $\mathrm{L}$ command is used to review the contents of these by typing 1 to 10 . Typing 11 calls up the data selected for fit described below. Typing 0 will cause a return to the list of commands.

The command C-CREATE DATA SET is used to enter data into one of the 10 available data sets. In response to the questions, choose the desired data set, the number of the point to be entered, and the title of that data point. Answering 0 will cause a return to the next higher level of command. Typing $\langle\mathrm{CR}\rangle$ as a title will cause a zero data point. This is the only way to zero data but it should be sufficient because data points can be selected or overwritten as desired.

The command S-SELECT DATA FOR FIT is used to assemble an array of 50 or fewer data points for the least squares fitting. in response to the questions, choose the desired data set and then the first and last point. For example, to choose points $1,3,4,5,6,8,9,10$, enter the successive commands, 1,1; 4,6; and 8,10 . To exit or to select from another data set, type 0,0 .

The command A-FIT TO A ONLY IN Y $=\mathrm{AX} /(1+\mathrm{BX})$ will cause the program to request that $\mathrm{B}$ be entered. Subroutine 9000 is then called to help select a starting value $A_{o}$ from the data set selected by $S$. The program will then proceed to the iterative fitting routine.

The command B-FIT TO B ONLY IN Y $=\mathrm{AX} /(1+\mathrm{BX})$ causes a similar response to that described above for $\mathrm{A}$.

The command F-FIT TO A AND B IN Y $=\mathrm{AX} /(1+\mathrm{BX})$ will cause the program to select starting values $A_{0}$ and $B_{0}$ and then proceed to the iterative fit.

A least-squares fit program seeks to minimize the chi-square

$$
x^{2}=\sum_{i} \frac{\left[Y_{i}-F\left(a, b, x_{i}\right)\right]^{2}}{a_{i}^{2}}=\sum_{i} Q_{i}^{2} .
$$


Expand $\mathrm{X}^{2}$ in a Taylor series about $\mathrm{a}=\mathrm{a}_{\mathrm{o}}+\Delta \mathrm{a}, \mathrm{b}=\mathrm{b}_{0}+\Delta \mathrm{b}$ :

$$
\begin{aligned}
\mathrm{x}^{2} & \left.\approx \sum \mathrm{Q}^{2}\right|_{0}+\left.\frac{\Sigma \delta Q^{2}}{\delta \mathrm{a}}\right|_{0} \Delta \mathrm{a}+\left.\left.\frac{\Sigma \delta Q^{2}}{\delta \mathrm{b}}\right|_{0}\right|_{\mathrm{b}}+\cdots \\
& \approx \sum Q^{2}+2 \Delta \mathrm{a} \sum \mathrm{Q} \frac{\delta Q}{\delta \mathrm{a}}+2 \Delta \mathrm{b} \sum \mathrm{Q} \frac{\delta Q}{\delta \mathrm{b}}+\cdots
\end{aligned}
$$

To minimize $\mathrm{X}^{2}$, set the first order derivatives of $\mathrm{X}^{2}, \delta \mathrm{X}^{2} / \delta \mathrm{a}$, and $\delta \mathrm{X}^{2} / \delta \mathrm{b}$, equal to zero:

$0=\sum Q \frac{\delta Q}{\delta a}+\Delta a \frac{\Delta \delta Q}{a} \frac{\delta Q}{a}+\frac{\Delta b \Sigma \delta}{a} \frac{\delta Q}{b}$

$0=\sum Q \frac{\delta Q}{\delta b}+\Delta a \Sigma \frac{\delta Q}{\delta b} \frac{\delta Q}{\delta a}+\Delta \frac{\delta Q Q}{\delta b} \frac{\delta Q}{\delta b}$

Converting to the notation of the program.

$0=s_{4}+a_{3} s_{1}+b_{3} s_{2}$,

and

$0=s_{4}+a_{3} s_{2}+b_{3} s_{3}$.

In matrix notation, this is

$\left(\begin{array}{c}-s_{4} \\ -s_{5}\end{array}\right)=\left(\begin{array}{c}s_{1} s_{2} \\ s_{2} s_{3}\end{array}\right)\left(\begin{array}{c}a_{3} \\ b_{3}\end{array}\right)$

or $\vec{F}=$ curvature matrix $\cdot \vec{a}$. The solution that must be iterated is

$a_{3}=\left(s_{2} s_{5}-s_{3} s_{4}\right) / D$,

$b_{3}=\left(s_{2} s_{4}-s_{1} s_{5}\right) / D$

$D=s_{1} s_{3}-s_{2} s_{2}$

The error matrix $=$ (curvature matrix $)^{-1}=\frac{1}{D}\left(\begin{array}{cc}s_{3} & -s_{2} \\ -s_{2} & s_{1}\end{array}\right)$.

From this we obtained (after convergence) the errors in the parameters

$\delta_{\mathrm{a}}=\sqrt{\mathrm{s}_{3} / \mathrm{D}}, \quad \delta_{\mathrm{b}}=\sqrt{\mathrm{s}_{1} / D}, \operatorname{cov}(\mathrm{a}, \mathrm{b})=-\mathrm{S}_{2} / \mathrm{D}$.

Other program variables are A2 and B2, which determine whether A or B is varied, the variance in each data point,

$G=\sigma_{1}=\sqrt{\sigma y^{2}+\frac{\delta f^{2}}{\delta x} \sigma x^{2}}$. 
and the chi-square-per-degree-of-freedom S6. If the assigned errors $\sigma x(i), \sigma y(i)$ correctly describe the variance in the data and if the choice of fitting function $\mathrm{F}$ is correct, the chi-square per degree of freedom will usually lie between 0.3 and 2.0 and thus provides a goodness-of-fit criterion. (Chi-square per degree of freedom will be 0 for a perfect fit, for example, a two-parameter fit to two data points). If the chi-square per degree of freedom is much larger than one, the errors have been underestimated. As a rough correction, increase $\sigma_{\mathrm{a}}$ and $\sigma_{\mathrm{b}}$ by the square root of chi-square per degree of freedom, and increase the covariance by the chi-square per degree of freedom.

After the above results are printed out, the data points used in the fit are listed. Next to each data point $Y_{i}$, the result $F\left(x_{i}, a, b\right)$ is also calculated. This listing provides a direct qualitative confirmation of the validity (or nonvalidity) of the fit. The corresponding error in $F$ is given by

$$
\delta F=\sqrt{\left(\frac{\delta F}{\delta x}\right)^{2} \sigma x^{2}+\left(\frac{\delta F}{\delta a}\right)^{2} \sigma a^{2}+\left(\frac{\delta F}{\delta b}\right)^{2} \sigma b^{2}-2 \frac{\delta F}{\delta a} \frac{\delta F}{\delta b} \operatorname{cov}(a, b)} .
$$

Note that the functions to be fit appear only in the subroutine at 9040 . To modify this program to fit other two-parameter functions, it is only necessary to insert new equations for $\mathrm{G}, \mathrm{Q}, \mathrm{Q} 1, \mathrm{Q} 2, \mathrm{~F}$, and F1.

\section{F. Additional Functions of MUBASIC}

A series of assembly language routines have been written by T. L. Atwell and compiled with MUBASIC. The resulting file is MUBASC, and it must be called whenever MUBASIC is to be used to address the CAMAC crate. The assembly language routines themselves are stored in a file called ADDLFN.MAC, which is available on N. Ensslin's master system disks. The most important routine is NAFD, which is used to send data or instructions between the computer and the CAMAC crate.

\section{FAST ELECTRONICS TUNE-UP}

\section{A. Phototube Assembly}

Examine each new tube to be sure its surface is clear and free of scratches. The interior of the tube should have a yellow tinge. If not, the gas has leaked out.

Push the tube gently onto the Ortec 265 base. Tape a 2-in.-wide piece of green felt around the center of the tube. This felt acts as a shock absorber and helps to maintain tube alignment.

Slide the tube into the mu-metal adapter and screw the adapter into place. Remove the outer cover from the tube base to expose the pots for gain adjustment.

\section{B. Phototube Gain Adjustment}

Before installation in the FNC, each PMT should be adjusted on the bench. This tune-up consists of setting the three pots on the Ortec 265 tube base for maximum gain.

Couple the tube to a plastic scintillator and place a source nearby. Make sure there are no light leaks. If the tube is coupled to an LRS 612 PMT amplifier, set the high voltage to about --2100 V. RCA 8575 or Amperex X.P-2230 tubes are suitable for the FNC. Because the Amperex tubes have about half of the gain of the RCA tubes, it is preferable to use six tubes of the same manufacture to make gain equalization easier to achieve. 
Connect the fast timing anode signal to the LRS 612 PMT amplifier, and look at the output with a fast oscilloscope. The output should be about 1-2 V in magnitude (Fig. 6-1), with about $50 \mathrm{mV}$ of noise. If there is an intense noise spectrum between 50 and $100 \mathrm{mV}$ (about one in four tubes seems to exhibit this), the tube should be rejected.

On the phototube base, adjust the two bleeder string controls, Focus (R/7) and Dynode (R 15), for maximum output signal. Adjust the green pot ( $R / 6)$, which is the voltage on the last dynode, for maximum output signal without pulse shape distortion. The adjustment of all three pots may require some iteration, because they are somewhat interrelated. Finally, use the green pot (R26) as an attenuator to roughly equalize the gain from all six phototubes.

\section{Phototube Installation}

Clean the face of the PMT and the mating surface on the plastic scintillator with Kleenex tissue dampened with ethanol. Other materials or solvents may damage the polished surfaces. Cover both cleaned surfaces with DOW DC-20-057 optical coupling compound. Screw the PMT assembly into place at the base of the scintillator. It is helpful to have someone else observe this procedure with a flashlight through the viewing ports at the top of the scintillator. The assembly should be tightened just enough to spread out the coupling compound, remove air bubbles, and give a clear view into the phototube. Both PMT assemblies on the same scintillator should be tightened together because pressure from one may loosen the other. When this is complete, tighten the lock nut on each assembly.

\section{Phototube Gain and Timing Adjustment}

After amplification by the LRS 612 amplifier, the PMT pulses should be 10-20 ns wide and range in size from noise level to 1-2 V. A HV setting of -2100 to $-2200 \mathrm{~V}$ will probably be required to obtain reasonable output pulses and reasonable coincidence response. This pulse spectrum is illustrated in Fig. 6-1. The final equalization of gain between the six tubes can be done now by displaying the pulse spectra two at a time on a fast oscilloscope. Overlap the two spectra very carefully, select NORMAL triggering, and then rapidly switch back and forth between them using the CHAN 1 and CHAN 2 display controls. The tube with the higher gain will show a more intense pulse spectrum. If adjustments need to be made, use the pots on the HV distribution box. Typically, several turns on the pots are required. If the range of the pots is not sufficient to equalize gains, readjust the green pots (R26) on the PMT bases or replace the weakest phototube.

Even if all cables from the PMTs to the circuit are of equal length, the output signals may not occur at exactly the same time because of variations in the transit times within the tubes (typically these times are

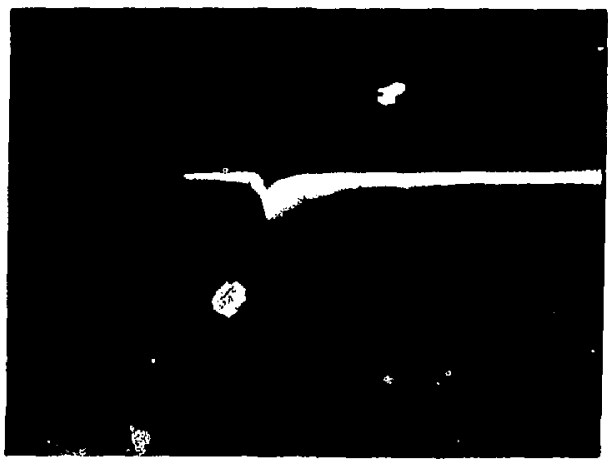

Fig. 6-1.

Photomultiplier tube output pulse-height spectrum (20 $\mathrm{ns} / \mathrm{cm}$ horizontal scale, $500 \mathrm{mV} / \mathrm{cm}$ vertical scale). 
25 to $40 \mathrm{~ns}$ ). For this reason it is necessary to check the relative timing of the phototubes. This is iest done at the output of the discriminators because these pulses all have the same shape and are the ones used for the fast timing circuits that follow.

First set the LRS 623 discriminators to $-100 \mathrm{mV}$ or whatever value has been chosen. This can be done using the test points on the front panel (output is 10x threshold). The settings can be checked conveniently by passing the amplifier output to the discriminator through the $1 \mathrm{M} \Omega$ scope input. Use the discriminator output as the trigger for the oscilloscope. Only those PMT pulses above the discriminator threshold will be visible on the scope. The discriminator output pulses should be $10 \mathrm{~ns}$ wide, $-800 \mathrm{mV}$ in cize.

Now check the relative timing of the two PMTs on each scintillator. This procedure may be aided by putting a strong coincidence source like ${ }^{60} \mathrm{Co}$ into the FNC cavity in addition to the random AmLi source already present, but this is not necessary. Pass the two discriminator outputs into one of the LRS 622 AND circuits through the $1 \mathrm{M} \Omega$ scope inputs. Use the AND output to trigger the scope. Because this trigger comes at the time of the later discriminator output, the leading edge of the earlier discriminator output will look fuzzy, and the leading edge of the later discriminator output will look sharp. For example, in Fig. 6-2 the upper discriminator output is about 2 ns ahead of the lower. Adjust the cable lengths between the PMT assemblies and the amplifiers until both leading edges look about equally fuzzy, as illustrated in Fig. 6-3. This procedure may be facilitated by overlapping both scope traces and switching rapidly back and forth between them. It should be possible to equalize the timing of signals from the two PMTs on each scintillator to within 0.5 to $1.0 \mathrm{~ns}$.

The above procedure is more difficult to use to check the relative timing between the three scintiliators. This timing was checked by setting a strong ${ }^{60} \mathrm{Co}$ source on the corner between two scintillators and checking the two nearest PMTs. A more complex procedure involves the use of a time-to-amplitude converter (TAC), a multichannel analyzer (MCA), and a delay box. The TAC should be used to look at the time differences between two scintillators. The LRS 622 AND coincidence signals from the two phototubes on each scintillator should be connected to the TAC. The TAC output should go into the ADC in the MCA. Calibrate the MCA to $1 \mathrm{~ns} / \mathrm{ch}$ annel by varying the time delay to the TAC stop and then adjusting the range of the TAC or ADC as needed. Set time $=0$ at the center of the MCA display by feeding one scintil:ator into two AND gates and then adjusting the time delay to the TAC stop or the MCA zero offset as needed. Now place a strong ${ }^{60} \mathrm{Co}$ source midway between two of the scintillators and look at the time difference with the TAC and the MCA. If the central peak is not centered at $t=0$, adjust both cable lengths from one scintillator relative to the other. Repeat this procedure with the other scintillator.

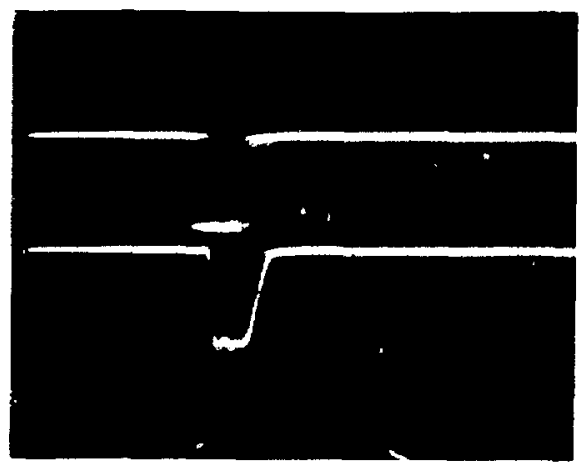

Fig. 6-2.

Discriminator output of two PMTs on one scintillator. The upper trace is abcve $2 \mathrm{~ns}$ ahead of the lower (10 $\mathrm{ns} / \mathrm{cm}$ horizontal scale, $500 \mathrm{mV} / \mathrm{cm}$ vertical scale).

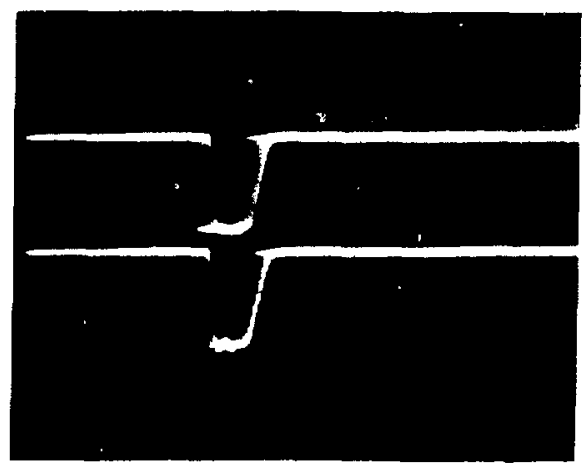

Fig. 6-3.

Discriminator output of two PMTs oin one scintillator. Both traces are simultaneous within $0.5 \mathrm{~ns}(10 \mathrm{~ns} / \mathrm{cm}$ horizontal scale, $500 \mathrm{mV} / \mathrm{cm}$ vertical scale). 


\section{E. Fast Timing Circuitry}

Complete the wiring of the fast electronics, as illustrated in Fig. 4-4. Keep all cable lengths as short as possible and all cables of similar function of equal length. The double and accidental double coincidence signals are clipped to $38.4 \mathrm{~ns}$ and $38.8 \mathrm{~ns}$, respectively. Other output pulse widths are given in Fig. 4-4. Terminators should be used where necessary to keep all output pulses at $-800 \mathrm{mV}$.

Check that immediately coincident events, such as (gamma,gamma) coincidences, will not be registered. The easiest way to do this is to feed only one scintillator into the triple OR and then check that the double coincidence module (top of the last LRS 622) has no output. Also, check the two inputs to the double coincidence module for correct amplitude, duration, and relative spacing. Correct timing is illustrated in Fig. 6-4.

A final confirmation of correct timing can be done by again using an MCA and a TAC connected between two scintillators. Use a strong source that emits gammas and neutrons in coincidence, and calibrate the MCA display as described earlier. Gate the input to the MCA with the double coincidence output of the fast electronics circuit. This makes it possible to check directly that the electronics acsept only events separated in time by -45 to -5 as or +5 to $+45 \mathrm{~ns}$. Within these windows the distribis on of time differences will be similar to that illustrated in Fig. 4-6. If the windows are not set correctly, adjust gate and delay lengths as needed.

The above cointrmation need not be carried out if the initial assays of background and random sources show that the real and accidental circuits are balanced at low and high rates, and if assays of fission sources show that the double and triple coincidence response is independent of background and linear with source strength.

\section{OPERATING INSTRUCTIONS}

\section{A. Sample Loading}

The sample holder mechanism is raised and lowered by the front Minarik controller located to the left of the FNC chassis. For the 4 in. high recovery cans, use the 3 in. spacer to center the can with respect to the scintillators and the neutron sources. Make sure that no other large samples of plutonium are in the glove box. Start the sample rotator motor with the switch mounted on the request assay box. Note that the request assay button is inoperative unless the sample is rotating. If the FNC software is in the Random Driver Available mode, a valid assay request will cause the terminal to respond with IS SAMPLE LOADED AND ROTATING?

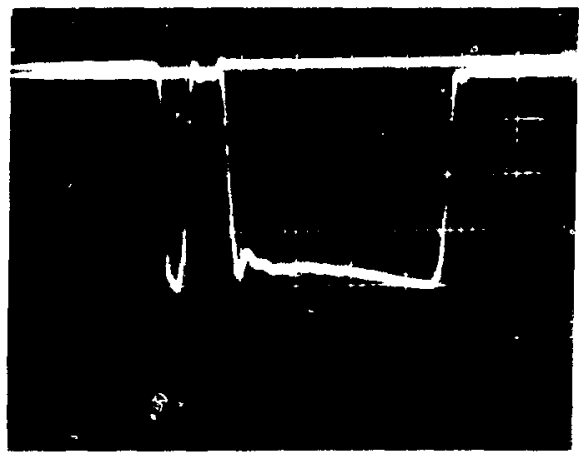

Fig. 6-4.

Correct timing at the input to the double coincidence module. One trace is a 5-ns-wide prompt signal; the other is a 40 -ns-wide delayed signal (10 ns/cm horizontal scale, $200 \mathrm{mV} / \mathrm{cm}$ vertical scale). 
B. Tl 743 Silent 700 Terminal Snttings

HI SPEED

FULL DUPLEX

ON LINE

NUM off

\section{Restarting Computer after Power Fail}

1. On the front panel of the computer, push CONTROL-HALT (push HALT while holding down CONTROL). Then push CONTROL-BOOT. The terminal should respond by printing four numbers and a $\$$.

2. Type DX $<C R>$ on the terminal. The terminal should respond with RT-11... and a . as a prompt.

3. Enter DAT DD-MON-YR $\langle\mathrm{CR}>$.

Enter TIM HH-MM-SS $\langle C R>$.

4. Type $R$ MUBASC $<C R>$. The terminal should respond with MU-BASIC... ENTER CONFIGURATION FILE...?

5. Type (letter) $O<C R>$. The terminal should respond with FILE--?

6. Type $1 \mathrm{UR}<\mathrm{CR}>$. The terminal should respond with READY.

\section{Turning off Power on CAMAC Crate}

If it is necessary to replace a module in the CAMAC crate without losing the computer memory, the following procedure should be followed.

1. Press CONTROL/HALT on computer.

2. Turn CAMAC crate offline.

3. Turn off crate power.

4. Loosen the screw at the bottom of the module and remove it.

5. Replace module, tighten screw with screwdriver.

6. Check to see that NIM - CAMAC adapter is all the way back into crate (NIM modules only).

7. Replace rear panel connectors (CAMAC modules only).

8. Turn on crate power.

9. Issue $\mathrm{C}$ and $\mathrm{Z}$ from crate controller by pressing INITIALIZE.

10. Turn crate online.

11. Press CONTROL/CONTINUE on computer.

This procedure will restore program operation, but of course the clock will not advance while the compute: " halted.

\section{E. Loading the Operating Software}

1. To start the assay program onc: IUBASIC is up (READY on terminal), type R DYMAC3.

2. The terminal will respond with IS FLOPPY DISK AVAILABLE FOR INITIALIZATION? Type YES $\langle$ CR $>$.

3. The terminal will respond with DO YOU WISH TO CHECK OR CHANGE INITIALIZATION? If you wish to review or change some of the contents of the virtual file VFl (100) that contains the calibration data, type $\mathrm{YES}\langle\mathrm{CR}\rangle$. If noi, type NO $\langle\mathrm{CR}\rangle$.

4. The terminal will respond with SOURCE IS DOWN, RANDOM DRIVER AVAILABLE. 


\section{F. Assay Instructions}

1. When the terminal has responded with RANDOM DRIVER AVAILABLE, and the sample is loaded and rotating, push the request assay button.

2. The terminal will respond with IS SAMPLE LOADED AND ROTATING? If so, answer YES $\langle\mathrm{CR}\rangle$.

3. The terminal will then request the desired counting time. Enter this in seconds.

4. The assay will now be started, and the assay number, date, and time will be printed. The remaining questions must be answered before the assay times out.

5. Enter operator's name.

6. Enter assay type. The operator response must be identical to one of the types listed. Some assay types will cause an active assay to be automatically perfurmed.

\begin{tabular}{|c|c|c|}
\hline Initials & Material & Active Assa \\
\hline BKG & background run & yes \\
\hline NOR & normalization standard & yes \\
\hline OXD & oxide & maybe \\
\hline FLU & fluoride & no \\
\hline PMP & electrorefined metal & no \\
\hline PRP & reduction metal (impure) & yes \\
\hline RES & electrorefining residues & yes \\
\hline
\end{tabular}

7. Enter sample identification, weight, diameter, and fill height if called for. This information does not affect the calculated mass of the sample, although the choice of assay type (item 6 a'jove) does.

8. Enter the enrichment (per cent ${ }^{240} \mathrm{Pu}$ ) as accurately as possible because this value is used to compute the total mass of plutonium.

9. The AUTOCYCLE question may be answered NO $<\mathrm{CR}>$ ( 1 run will occur), YES $\langle\mathrm{CR}\rangle(100$ runs will occur), $N>C R<$ ( $N$ runs will occur), or just $\langle\mathrm{CR}\rangle$ (1 run will occur).

10. If a question has been answered incorrectly, answer "B" on the next question to backspace.

11. If a question has been answered with incorrect syntax, and the terminal responds with STOP AT LINE N, type GO TO $\mathrm{N}$ to repeat the question and continue.

12. To stop a run before it times out, type CONTROL/C. The terminal will respond with READY. Then type GO TO 1000. The terminal will respond with RANDOM DRIVER AVAILABLE.

\section{G. Interpretation of Assay Print-out}

Table VII-1 illustrates the print-out obtained from the passive and active assay of PRP73, a sample of reduction metal. At that time the assay length was fixed at $500 \mathrm{~s}$. In other respects the computer print-out is the same today. 
TABLE VII-1

SAMPLE PRINT-OUT

RANDOM DRI VER AVAILABLE

IS SAMPLE LOADED AND ROTATING

PASSIVE ASSAY NO.

Y

OPERATOR NAME

BKG, CAL, OXD, FLU, PMP, PRP, RES

OK

SAMPLE ID, COMMENTS

SAMPLE WGT (GMS)

SAMPLE DIAM (CM)

FILL HEIGHT (CM)

ENRICHMNT (240)

AUTOCYCLE

PRP

PRP73

600.7

7.5

1.5

5.84

N

TIME HE 3 DOUBLES ACC DBL TRIPLES ACC TRP $\begin{array}{llll}500 & 212 & 204988 & 2083\end{array}$

5511

LEFT MIDD

RIGHT

$\begin{array}{ccc}\text { ROOM TEMP } & \text { BACKGROUND } & \text { SINGLES } \\ 22.4 & 4.067 & 12245 \pm 4.948 \\ \text { CRATE TEMP } & \text { TEMP CORR } & \text { FLUX RATIO } \\ 35.7 & 1.011 & 1 \pm 0 \\ \text { PMT TEMP } & \text { MATL TEMP } & \text { FLUX CORR } \\ 23.6 & 16.99 & 1 \pm 0 \\ \text { HV TEMP } & \text { PLUT DENS } & \text { ALPHA } \\ 22.4 & 19.52 & 0 \pm 0\end{array}$

$\begin{array}{llll}6 & 1.780+06 & 2.418+06 & 1.924+06\end{array}$

TRIPLES

DOUBLES

$11.01 \pm 0.14 \quad 405.81 \pm 0.9101$

TRIPLES/DOUBLES CORR.DOUBLES

$0.026 \pm 3.04232 \pm 2.151$

LEAKAGE MULT NORMALIZATION

$1.22 \pm 4 .-03 \quad 0.977 \neq 8 .-03$

SELF-MULT CORR ASSAY IN GMS PU240

$1.73 \pm 0.015 \quad 41.99 \pm 0.57$

SOURCE IS UP

ACTIVE ASSAY NO. $\quad 17$ STARTED AT 22/NOV/78-09:27

TIME HE3 DOUBLES ACC DBL TRIPLES ACC TRP LEFT MIDD RIGHT

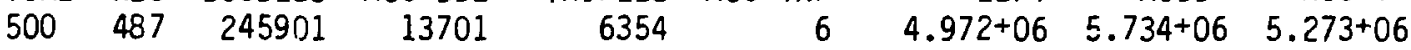

\begin{tabular}{cccccc} 
ROOM TEMP & BACKGROUND & SINGLES & TRIPLES & DOUBLES \\
22.6 & 5.9 & $31959 \pm 7.994$ & $12.69 \pm 0.14$ & $464.4 \pm 1.019$ \\
CRATE TEMP & TEMP CORR & FLUX RATIO & TRIPLES/DOUBLES & CORR. DOUBLES \\
35.8 & 1.011 & $0.954 \pm 0.10$ & $0.029 \pm 3 .-03$ & $34.72 \pm 0.898$ \\
PMT TEMP & MATL TEMP & FLUX CORR & LEAKAGE MULT & NORMALIZATION \\
23.4 & 16.99 & $0.990 \pm 0.02$ & $1.22 \pm 4 .-03$ & $0.977 \pm 8 .-03$ \\
HV TEMP & PLUT DENS & \multicolumn{2}{c}{ ALPHA } & SELF MULT CORR ASSAY IN GMS PU240 \\
22.4 & 15.31 & $0 \pm 0$ & $1.63 \pm 0.133$ & $508.33 \pm 15.37$
\end{tabular}

FINAL RESULT FOR TOTAL PLUTONIUM: $541.26 \pm 16.36$ GMS

SOURCE IS DOWN

RANDOM DRIVER AVAILABLE 
The first line is the raw data received from the CAMAC scalers.

TIME

HE3

DOUBLES

ACC DBLS

TRIPLES

ACC TRP

LEFT

MIDD

RIGHT

Assay length in seconds. This is the measured length, and may exceed the requested length by a few seconds.

Total neutron counts from ${ }^{3} \mathrm{He}$ tubes. The circuit is now turned off Counts from double coincidence circuit.

Counts from accidental double coincidence circuit.

Counts from triple coincidence circuit.

Counts from accidental triple coincidence circuits.

Total counts from left scintillator AND circuit.

Total counts from middle scintillator AND circuit.

Total counts from right scintillator AND circuit.

Below this line, the data are roughly organized in columns.

ROOM TEMP

CRATE TEMP

PMT TEMP

HV TEMP

BACKGROUND

TEMP CORR

MATL DENS

PLUT DENS

SINGLES

FLUX RATIO

FLUX CORR

ALPHA

TRIPLES

TRIPLES/DOUBLES

LEAKAGE MULT

SELF-MULT CORR
Temperature in ${ }^{\circ} \mathrm{C}$ at top of the electronics cabinet.

Temperature in ${ }^{\circ} \mathrm{C}$ at back of the CAMAC crate.

Temperature in ${ }^{\circ} \mathrm{C}$ at base of one of the photomultiplier tubes.

Temperature in ${ }^{\circ} \mathrm{C}$ at the top of the $\mathrm{HV}$ power supply.

Passive or active double coincidence background. These values are updated by each BKG run. The triple coincidence background values are also stored and updated, but are not printed out.

The temperature correction is calculated as described in Sec. V.B, but it is not applied to the data.

Sample weight (pi $\times$ sample diameter $\times$ sample fill height), as supplied by the operator.

For passive assays, this is the grams of ${ }^{240} \mathrm{Pu}$ determined by the assay/(enrichment $\times$ sample volume). For active assays, this is the grams of ${ }^{239} \mathrm{P} 1 \mathrm{a}$ determined by the assay/[(1 enrichment) $\times$ sample volume].

(LEFT + MIDD + RIGHT)/TIME, total count rate.

${ }^{3} \mathrm{He}$ neutron count rate during active assay

less ${ }^{3} \mathrm{He}$ neutron count rate during passive assay, divided by a reference rate determined during the last active background update.

A correction for neutron thermalization within the sample as determined by the flux ratio. The equation is given in Sec. V.B. This correction is not applied to the measured response.

The ratio of neutrons from (alpha,n) reactions to neutrons from spontaneous fission in the sample that was assumed for the self-multiplication correction applied to this assay.

(TRIPLES - ACC TRP)/TIME, triples count rate.

M.easured triples rate less triples

background divided by measured doubles rate less

doubles background.

Net leakage multiplication within the sample

as calculated by the self-multiplication correction.

The self-multiplication correction applied

to this assay. The correction is described in Sec. V.B. 
DOUBLES

CORR. DOUBLES

NORMALIZATION

ASSAY IN GMS

PU240 (PU239)

FINAL RESULT FOR TOTAL PU
(DOUBLES - ACC DBL)/TIME,

double coincidence rate.

The above doubles rate less the doubles

background, divided by the self-multiplication

correction. For active assays, the passive duubles rate

less its background is also subtracted before the

self-multiplication correction is applied.

The measured double coincidence response

is adjusted by this factor, which is determined by

counting standard No. 9 as a NORM run. The NORM

run updates the passive and active normalization

factors whenever it is performed.

The corrected double coincidence rate

$x$ the normalization constant is used

to compute the mass from the relation $A \times$ response/

$\left(1+B^{*}\right.$ response), where $A$ and $B$ are calibration

constants.

If the enrichment was specified, convert

the passive (and active if done) assay to

grams of total plutonium. Use the passive assay as the

final result unless the active assay is smaller by more

then 1 std dev.

\section{H. Measurement Control Procedures}

It is strongly recommended that a BACKGROUND (BKG) (first) and a NORMALIZATION (NORM) run (second) be performed before unknown samples are assayed. This procedure should be carried out every day that the FNC is used. If the amount of nuclear material in the vicinity of the FNC changes appreciably during the day, a new BKG run should be done immediately. If there is any doubt about the proper operation of the instrument, the NORM run should be repeated.

The BKG run updates the passive and active double and triple coincidence backgrounds stored in the computer. The NORM run updates the passive and active double coincidence normalization factors. Both runs together allow the measured response to be corrected properly for room background variations and instrument drift. If these runs are not carried out regularly, the software can be modified to make them mandatory.

\section{Calibration Procedures}

1. Complete the electrical tune-up and timing adjustment of all circuits (Sec. VI).

2. Carry out overnight BKG and NORM runs to ensure that the measured backgrounds and responses are of reasonzitic magnitude and are free of strong drifts and high voltage breakdowns.

3. Run a large, very hot sample to ensure that the circuitry is balanced at very high count rates.

4. If a self-multiplication correction is to be used as a correction factor or diagnostic check, assay a small (20 g or less) sample of pure electrorefined metal overnight to get good statistics. Perform long, careful BKG runs before and after. Determine the triples/doubles ratio from this "nonmultiplying" sample and store it in the floppy disk file. 
5. For each material category, assay a series of standards covering the desired range of sample sizes. Perform multiple assays to get gooci statistics and better reliability. Perform frequent BKG and NORM runs.

6. For each material category, carry out a least-squares fit of (double coincidence response less coincidence background) times normalization divided by self-multiplication correction (if desired) as a function of ${ }^{210} \mathrm{Pu}$ effective mass and/or ${ }^{239} \mathrm{Pu}$ effective mass. Load $\mathrm{A}, \sigma \mathrm{A}, \mathrm{B}, \sigma B$, and the covariance values into the floppy disk file.

\section{MAINTENANCE}

\section{A. List of Manuals Supplied}

Table VIII- 1 is a list of equipment manuals supplied with the FNC. These manuals cover all of the hardware except the Power Designs 3K-40 HV Power Supply.

TABLE VIII- 1

LIST OF MANUALS SUPPLIED

\begin{tabular}{ll}
\hline 1. SEC 1510A/12 & CAMAC power supply \\
2. SEC DCC-11 & CAMAC crate controller \\
3. SEC WW-004 & Prototype module \\
4. LRS 2551 & 12-channel scaler \\
5. KS 3290 & Dataway display \\
6. KS 3530 & Relay multiplexer \\
7. KS 3552-1 & Optically isolated ADC \\
8. ByRa 5601 & TTL-NIM converter \\
9. LRS 365 AL & Dual 4-fold logic \\
10. LRS 622 & Quad 2-fold logic \\
11. LRS 623 & Octal discriminator \\
12. LRS 612 & 12-channel PMT amplifier \\
13. Canberra 3105 HV powe- supply & \\
14. Canberra 2105 amplifier-SCA & \\
15. DEC RX01 & Floppy disk \\
16. DEC RX01 & Floppy disk engineering drawings \\
17. DEC PDP11/04 & Engineering drawings \\
18. DEC DL11-W (M7856) & Serial line interface \\
19. DEC M9301 & Bootstrap/terminator \\
20. DEC KY11-LB & Keyboard interface \\
21. TI 743 & Printer \\
22. Variac 1592 ac regulator & \\
23. Minarik motor controller & \\
24. Bodine NSH-34RJ motor & \\
25. Ortec Inc. 265 PMT base & \\
\hline
\end{tabular}




\section{B. Routine Maintenance}

1. The intake air filter on the electronics cabinet should be replaced every 6 months.

2. The TI 743 Printer should receive a new ribbon and lubrication every 6 to 12 months.

3. Every 12 months, the PMTs should be inspected to see if the optical coupling compound between the tube face and the scintillator has separated. The FNC chassis should be disconnected from its back frame and rolled forward. Remove the chassis cover and the black tape over the scintillators and look down at the phototubes. CAUTION: The PMT HV must be off when the scintillators are exposed to light.

\section{Troubleshooting}

When unusual assay results or warning messages indicate equipment malfunction, it is usually possible to localize the area of trouble by looking at the data print-out.

1. If the trouble is in the fast electronics modules (which is often the case), it is easy to locate and replace the faulty module by using a fast oscilloscope to look at output signals.

2. If assay results are not reproducible or if the normalization factor varies significantly from day to day, the PMT HV power supply may be out of regulation. This can be checked by using a strip chart recorder to monitor the voltage.

3. Failure of a PMT or failure of a PMT to scintillator coupling is usually indicated by a decreasing response. an increasing normalization factor, an imbalance in the count rates between scintillator,s, and/or a decrease in assay precision due to a decrease in response from the affected scintillator.

4. Repeated messages of SOURCE IS STALLED usually indicate failure of the motor controller relays, damage to the microswtiches, or that the sources have not reached their destination in the time allowed by the computer.

\section{Anticipated Problem Areas}

The problem or providing a proper normalization for active assays is not yet solved. Because active assays have a lower statistical precision than passive assays, it is desirable to make the active normalization vary with the passive normalization. However, a large change in phototube high voltage or in the ratios of responses between scintillators seems to affect the active normalization more than the passive normalization. At present, the active normalization is set to be $90 \%$ of the passive normalization, but this ratio should be checked or changed whenever a large change in phototube response occurs.

\section{REFERENCES}

1. F. X. Hass, J. L. Lawless, W. E. Herren, and M. E. Hughes, "Measurement of Plutonium and Americium in Molten Salt Residues," in "Measurement Technology for Safeguards and Materials Control," National Bureau of Standards Special report 582 (June 1980).

2. "Nuclear Safeguards Research and Development, Program Status Report, January-April 1978," Los Alamos Scientific Laboratory report LA-7439-PR (December 1978), p. 30. 
3. L. V. East and J. E. Swansen, "Charge Sensitive Preamplifier for Use with Large Proportional Counter Assays," Los Alamos Scientific Laboratory report LA-4972-MS (June 1972).

4. J. L. Sapir, Comp., "Nuclear Safeguards Research and Development Program Status Report, September-December 1977," Los Alamos Scientific Laboratory report LA-721 I-PR (July 1978), p. 14.

5. N. Ensslin, J. Stewart, and J. Sapir, "Self-Multiplication Correction Factors for Neutron Coincidence Counting," Inst. Nucl. Mater. Manage. 8 (1979), p. 60, Eqs. (13-24). 